\title{
TITLE: Robust three-dimensional expansion of human adult alveolar stem cells and SARS-CoV-2 infection
}

\author{
Authors \\ Jeonghwan Youk $(1,2,12)$, Taewoo Kim $(1,12)$, Kelly V. Evans $(3,4,12)$, Young-II Jeong $(5,12)$, \\ Yongsuk Hur (6,12), Seon Pyo Hong (7,12), Je Hyoung Kim (5), Kijong Yi (1), Su Yeon Kim (1), Kwon \\ Joong $\mathrm{Na}(8)$, Thomas Bleazard (9), Ho Min Kim $(1,10)$, Natasha Ivory (11), Krishnaa T. Mahbubani \\ (11), Kourosh Saeb-Parsy (11), Young Tae Kim $\left(8,{ }^{*}\right)$, Gou Young Koh $\left(7,{ }^{*}\right)$, Byeong-Sun Choi $\left(5,{ }^{*}\right)$, \\ Young Seok Ju $\left(1,2,{ }^{*}\right)$ and Joo-Hyeon Lee $\left(3,4,{ }^{*}\right)$
}

\section{Affiliations}

1. Graduate School of Medical Science and Engineering, Korea Advanced Institute of Science and Technology, Daejeon 34141, Republic of Korea

2. GENOMEINSIGHT Inc., Daejeon 34051, Republic of Korea

3. Wellcome-MRC Cambridge Stem Cell Institute, Jeffrey Cheah Biomedical Centre, University of Cambridge, Cambridge, CB2 AOW, United Kingdom

4. Department of Physiology, Development and Neuroscience, University of Cambridge, Cambridge, CB2 3EL, United Kingdom

5. Division of Viral Disease Research, Center for Infectious Diseases Research, Korea National Institute of Health, Korea Centers for Disease Control and Prevention, Cheongju 28159, Republic of Korea

6. BioMedical Research Center, Korea Advanced institute of Science and Technology, Daejeon 34141, Republic Korea

7. Center for Vascular Research, Institute for Basic Science, Daejeon 34126, Republic of Korea 8. Department of Thoracic and Cardiovascular Surgery, Seoul National University Hospital, Seoul 03080, Republic of Korea

9. The National Institute for Biological Standards and Control, Blanche Lane, South Mimms, Potters Bar, Hertfordshire EN6 3QG, United Kingdom 
10. Center for Biomolecular and Cellular Structure, Institute for Basic Science, Daejeon 34126,

Republic of Korea

11. Department of Surgery and Cambridge NIHR Biomedical Research Centre, Biomedical Campus, University of Cambridge, Cambridge, CB2 2QQ, United Kingdom

12. These authors contributed equally

\section{* Correspondence to}

Young Seok Ju, ysju@kaist.ac.kr, +82-42-350-4237, Associate Professor, KAIST, Daejeon 34141, Republic of Korea

Joo-Hyeon Lee, jhl62@cam.ac.uk, Group Leader, Wellcome-MRC Cambridge Stem Cell Institute, University of Cambridge, Cambridge CB2 AOW, United Kingdom

Byeong-Sun Choi, byeongsun@korea.kr, Division head, Division of Viral Disease Research, Center for Infectious Disease Research, Korea Centers for Disease Control \& Prevention, Cheongju, Republic of Korea

Gou Young Koh, gykoh@kaist.ac.kr, Director, Center for Vascular Research, Institute of Basic Science, Daejeon 34126, Republic of Korea

Young Tae Kim, ytkim@snu.ac.kr, Professor, Department of Thoracic Surgery, Seoul National University Hospital, Seoul 03080 , Republic of Korea 


\section{Abstract}

Severe acute respiratory syndrome-coronavirus 2 (SARS-CoV-2), which is the cause of a present global pandemic, infects human lung alveolar cells (hACs). Characterising the pathogenesis is crucial for developing vaccines and therapeutics. However, the lack of models mirroring the cellular physiology and pathology of hACs limits the study. Here, we develop a feeder-free, long-term threedimensional (3D) culture technique for human alveolar type 2 (hAT2) cells, and investigate infection response to SARS-CoV-2. By imaging-based analysis and single-cell transcriptome profiling, we reveal rapid viral replication and the increased expression of interferon-associated genes and proinflammatory genes in infected hAT2 cells, indicating robust endogenous innate immune response. Further tracing of viral mutations acquired during transmission identifies full infection of individual cells effectively from a single viral entry. Our study provides deep insights into the pathogenesis of SARSCoV-2, and the application of long-term 3D hAT2 cultures as models for respiratory diseases.

Keywords: lung alveolar type 2 cells; SARS-CoV-2; COVID-19; co-culture; virus; organoid; transcriptome; pathogenesis; single-cell RNA-seq; interferon 


\section{Main}

Several members of the family Coronaviridae are transmitted from animals to humans and cause severe respiratory diseases in affected individuals ${ }^{1}$. These include the severe acute respiratory syndrome (SARS) and the Middle East respiratory syndrome (MERS) coronavirus. Currently, Coronavirus disease 2019 (COVID-19), caused by severe acute respiratory syndrome coronavirus 2 (SARS-CoV-2), is spreading globally ${ }^{2}$ and more than 11.8 million confirmed cases with $~ 542 \mathrm{~K}$ deaths have been reported worldwide as of $7^{\text {th }} \mathrm{Jul} 2020^{3}$. The lung alveoli are the main target for these emerging viruses ${ }^{4}$.

To develop strategies for efficient prevention, diagnosis, and treatment, the characteristics of new viruses, including mechanisms of cell entry and transmission, kinetics in replication and transcription, host reactions and genome evolution, should be accurately understood in target tissues. Although basic molecular mechanisms in SARS-CoV-2 infection have been identified ${ }^{5-8}$, most findings have been obtained from experiments using non-physiological cell lines ${ }^{9}$, model animals, such as transgenic mice expressing human angiotensin-converting enzyme $2\left(\right.$ ACE2) ${ }^{10}$, ferrets ${ }^{11}$ and golden hamsters ${ }^{12}$, or from observation in clinical cohorts ${ }^{13}$ and/or inference from in-silico computational methods $^{14-16}$. As a consequence, we do not fully understand how SARS-CoV-2 affects human lung tissues in the physiological state.

Development of three-dimensional (3D) stem cell-derived organotypic culture models, conventionally called organoids, has enabled various physiologic and pathological studies using human-derived tissues in vitro ${ }^{17-19}$. Organoid models established from induced pluripotent stem cells (iPSCs), or adult stem cells in the human kidney, intestine, and airway have been used to investigate SARS-CoV-2 pathogenesis ${ }^{20-23}$. Although human alveolar type 2 cells (hereafter referred to as hAT2s) are believed to be the ultimate target cells for SARS-CoV-2, their infection model has not previously been introduced.

\section{Long-term 3D culturing of hAT2s without mesenchymal niche}

We have established feeder-free, 3D hAT2 organoids (hereafter referred to as hAOs; definition of organoid is available at ref. ${ }^{24}$ ) with defined factors which support molecular and functional identity of 
hAT2 cells over multiple passages, showing substantial improvements from the previous application of co-culture models ${ }^{25-27}$. Briefly, single-cell dissociated hAT2 cells were isolated by fluorescenceactivated cell sorting (FACS) for the hAT2 surface marker HTII-280 (CD31-CD45-EpCAM+HTII280+) ${ }^{25,28}$ (Fig. 1a; Extended Data Fig. 1a). Isolated HTII-280+ cells showed higher expression of AT2 cell marker SFTPC, while HTII-280- cells revealed higher expressions of basal cell marker TP63 and secretory cell marker SCGB1A1 (Extended Data Fig. 1b). We then plated HTII-280+ hAT2 cells into Matrigel for 3D cultures with our expansion medium, supplemented with CHIR99021, RSPO1 (Rspondin 1), FGF7, FGF10, EGF, NOG (Noggin), and SB431542, that are known to support the growth of human embryonic lung tip cells ${ }^{29}$. HTII-280- cells were also cultured under conditions supporting human bronchial (airway) organoids (hereafter referred to as hBOs) that have previously been reported ${ }^{30}$. hAOs established from single hAT2 cells grew up to 4 weeks with heterogeneous morphology including budding-like and cystic-like structures consisting of mature AT2 cells expressing pro-SFTPC, HTII-280, and ABCA3, as well as exhibiting uptake of Lysotracker, a fluorescent dye that stains acidic organelles such as lamellar bodies ${ }^{25}$ (Fig. 1b and 1c). In contrast, hBOs grew quickly by day 14 with cystic-like structures consisting of a number of airway cell types, including KRT5+TP63+ basal cells and SCGB1A1+ secretory cells, as previously reported ${ }^{30}$ (Fig. 1d and 1e). WNT activation was identified as an essential factor for $\mathrm{hAO}$ formation, because no colony formation was found in the absence of WNT activator CHIR99021 in culture (Extended Data Fig. 1c). Importantly, our culture system allows the long-term expansion (>10 months) of hAT2s, although colony forming efficiency varied between tissue samples and reduced at later passages (Extended Data Fig. 1d). Over passaging via single cells, hAT2s consistently formed organoids, exhibiting SFTPC expression following 9 months of continuous cultures, although growth began to slow, as evident by reduced organoid size and lower forming efficiencies (Extended Data Fig. 1d and 1e). Alveolar type 1 cells (hAT1s) expressing HOPX and PDPN were also observed during early cultures, demonstrating differentiation capacity of hAT2 cells in our hAOs (Extended Data Fig. 1f), although later passages exhibited loss of these cells.

\section{Robust infection of SARS-CoV-2 into hAOs}

The expressions of ACE2 and TMPRSS2, which are necessary for SARS-CoV-2 infection, were observed in the membrane and cytoplasm of hAO cells (Fig. 1f and 1g; Extended Data Fig. 1g). We 
next infected hAOs and hBOs with SARS-CoV-2 at a multiplicity of infection (MOI) of 1 . The viral particles were prepared from a patient (known as KCDC03) who was diagnosed with COVID-19 on $26^{\text {th }}$ Jan, 2020, after traveling to Wuhan, China ${ }^{31}$. Vero cells were also infected as a positive control, although this was not directly comparable to our 3D models due to different technical procedures.

Infectious virus particles increased to significant titers in hAOs (Fig. 1h-1k; Extended Data Fig. 2), reaching maximum levels within the 1st day post infection (dpi), suggesting that full infection occurs within 1 day from viral entry to hAOs. In hBOs, the increment of viral particles was observed as consistent with another study ${ }^{32}$, but their titers were $<100$ times lower than hAOs (Fig. $\mathbf{1 h}$ and $\mathbf{1 i}$;

Extended Data Fig. 2). In line with viral particles, the amount of the viral RNA in hAOs and in its culture supernatant reached a plateau at $1 \mathrm{dpi}$ (Fig. 1j and 1k). Although infected Vero cells exhibited significant cytopathic effects at $1 \mathrm{dpi}$, typically cell rounding, detachment, degeneration and syncytium formation ${ }^{9}$, SARS-CoV-2 infected hAOs and hBOs did not show prominent macroscopic pathologies up until $10 \mathrm{dpi}$.

\section{Structural changes of hAOs to SARS-CoV-2 infection}

Immunostaining for double-stranded viral RNA (dsRNA) and nucleocapsid protein (NP) of SARSCoV-2 identified widespread viral infection in hAT2 cells co-expressing pro-SFTPC and ACE2 in hAOs (Fig. 2a and 2b; Extended Data Fig. 3). To further determine subcellular events at a higher resolution, transmission electron microscopic analysis was performed at 2 dpi (Fig. 2c-2l; Extended

Data Fig. 4). Most cells ( $80 \%)$, containing secretory vesicles and surfactant proteins (lamellar bodies $^{25}$ ), showed discernible viral particles in the cytoplasm. A fraction of cells in hAOs showed much higher viral burdens than other cells, with as many as 500 copies in the $100 \mathrm{~nm}$ section, implying that $>10,000$ SARS-CoV-2 particles per cell. Aggregated viral proteins, which appeared as electron-dense regions near nuclei ${ }^{33}$, were also detected (Fig. 2c). Accordingly, several key pathogenic phenotypes were observed in the infected hAOs. Alveolar cells with enormous vacuoles were frequently observed (Fig. 2c, 2f, 2h, 2i), similar to cytopathic signals in Zika virus ${ }^{34}$. Doublemembrane vesicles (DMVs), subcellular structures known as viral replication sites frequently seen in the early phase of infection ${ }^{35,36}$, were observed in the vicinity of zippered endoplasmic reticulum in a small fraction of hAO cells (Fig. 2j and 2k). Viral particles were dispersed in the cytosol (Fig. 2e) or 
enclosed in the small vesicular structures (Fig. $\mathbf{2 f}$ and 2i). Diverse forms of viral secretion were also observed mainly through the apical surfaces of hAT2 cells (Fig. $\mathbf{2 g}$ and $\mathbf{2 l}$ ). More ultrastructural pathologies are available at Extended Data Fig. 4 and the EMPIAR data archive (see Data availability).

\section{Transcriptional changes of hAOs to SARS-CoV-2 infection}

From strand-specific deep RNA-sequencing, we explored gene expression changes in the infected hAOs. Indeed, a set of human genes were differentially expressed as infection progressed (i.e., 0, 1 and 3 dpi), although most genes showed good correlations (Extended Data Fig. 5a and

Supplementary Table 1). Cytokeratin genes (including KRT16, KRT6A, KRT6B, and KRT6C), genes involved in keratinization (including SPRR1A), cytoskeleton (including S100A2) and cell-cell adhesion genes (including DSG3), were significantly reduced to $\sim 2-3 \%$ in hAOs at $3 \mathrm{dpi}$ (Fig $3 \mathbf{a}$ and $\mathbf{3 b}$ ). Many more genes were upregulated in the infected hAOs specifically at $3 \mathrm{dpi}$. In particular, transcription of a broad range of interferon-stimulated genes (ISGs), known to be typically activated by type I and III interferons ${ }^{37}$, were remarkably increased (Fig. 3a and $\mathbf{3 b}$ ). These genes include interferon induced protein genes (such as IFI6, IFI27, IFI44, IFI44L), interferon induced transmembrane protein genes (such as IFITM1), interferon induced transmembrane proteins with tetratricopeptide repeats genes (IFIT1, IFIT2, IFIT3), 2'-5'-oligoadenylate synthetase genes (OAS1, OAS2), and miscellaneous genes known to be involved in innate cellular immunity (MX1, MX2, RSAD2, ISG15). These genes were expressed to $>20$ times higher levels in hAOs at 3 dpi than at 0 dpi. Many other known ISGs also showed moderate inductions (2-20 times) at 3 dpi, including BTS2 ( 15 times), OAS3 ( 11 times), HERC5 ( 15 times), HERC6 ( 12 times) and USP18 ( 12 times). Antiviral functions are known for these ISGs ${ }^{38}$ including (1) inhibition of virus entry (MX genes, IFITM genes), (2) inhibition of viral replication and translation (IFIT genes, OAS genes, ISG15, HERC5, HERC6, USP18) and (3) inhibition of viral egress (RSAD2 and BST2). Of note, given that immune cells are absent in our culture system, the innate immune response was completely autologous to alveolar cells, mimicking the initial phase of SARS-CoV-2 alveolar infection. In line with the notion, innate induction of some type I and type III interferons was observed. Of the 20 interferon genes, an interferon beta gene (IFNB1) and three interferon lambda genes (IFNL1, IFNL2, and IFNL3) showed significant transcriptional induction, although their absolute changes were not substantial (Fig. 3c). The surface 
receptors of interferons were stably expressed in hAO cells without reference to viral infection (Fig.

3c). Downstream signalling genes of the receptors were also upregulated such as STAT1 $(\sim 3.5$ times), STAT2 ( 2.5 times) and their associated genes IRF1 ( 2.4 times) and IRF9 ( 6.4 times). Of note, IRF1 is known to be specific to type I interferon responses ${ }^{39}$, while type I and type III ISGs are generally overlapping ${ }^{40}$.

In addition to ISGs, genes in the viral sensing pathway in cytosol showed increased expression in the infected hAOs at $3 \mathrm{dpi}$, for example, DDX58 (official gene name of RIG-1, from 1.9 to 25.0 TPM), IFIH1 (also known as MDA5, from 5.8 to 30.3 TPM), and TLR3 (Toll-like receptor 3, from 1.3 to 3.7 TPM), IRF7 (Interferon regulatory factor 7, from 4.4 to 29 TPM) and IL6 (0.6 to 2.5 at $1 \mathrm{dpi}$; proinflammatory factor).

Notably, these transcriptional changes were much stronger in hAOs than in hBOs. In the similar transcriptome profiling of the infected hBOs, the genes aforementioned were not significantly altered (Supplementary Table 2 and Extended Data Fig. 5b). In addition, we identified few hBO specific differentially expressed genes (Extended Data Fig. 5c). This finding implies cellular tropism of SARSCoV-2 viral infection.

\section{Expression of SARS-CoV-2 genes in the infected organoids}

We further analysed the viral RNA sequences obtained from the infected models. In agreement with the plaque assay (Fig. 1h and 1i), relative transcription of SARS-CoV-2 genes plateaued by $1 \mathrm{dpi}$ (Fig. 3d), which is earlier than the host gene expression changes. Approximately $50 \%$ of the RNA sequencing reads were mappable to the SARS-CoV-2 genome in hAOs from 1 dpi (Fig. 3d), indicating prevailing viral gene expression in infected hAO cells as observed in Vero cells ${ }^{6}$. Of note, the proportion of viral transcripts was much lower in the infected hBOs.

Transcripts from SARS-CoV-2 were not mapped uniformly to the viral genome sequence, but 3' genomic regions, where canonical subgenomic RNAs are located, showed much higher read-depth in all samples, consistent with the previous report ${ }^{6}$ (Fig. 3e). The vast majority of viral RNA sequences 
produced from the infected hAOs and hBOs was in the orientation of positive-sense RNA strands (Fig. 3e; for example, $99.98 \%$ vs $0.02 \%$ for positive- and negative-sense RNAs, respectively, from hAO at $1 \mathrm{dpi})$. This is in good agreement with the nature of SARS-CoV-2, which is an enveloped, nonsegmented, and positive-sense RNA virus.

By cross-comparison of viral RNA sequences produced from a total of 11 infected hAOs $(n=5)$ and hBCs $(n=6)$, we identified 20 viral base substitutions (Supplementary Table 3). No mutation was at $100 \%$ variant allele fraction (VAF) and exclusive to an infected sample. Instead, sequence alterations showed a broad range of quasispecies heterogeneity in each culture (VAF ranges from $0.1 \%$ to $73.1 \%$; Fig. 3f), and a large proportion of the mutations ( $n=16 ; 80 \%)$ were shared by two or more infected models (by the cut-off threshold of $0.1 \%$ ). Therefore, we speculate that most of these sequence changes were originally present in the pool of viral particles before their inoculation. Given the fact that these viral particles were prepared from one of the earliest COVID-19 patients, our finding suggests that mutations can accumulate in the viral genomes in a small number of rounds of viral transmissions, and appear with dramatic changes in quasispecies abundance. A substantially higher proportion of specific mutations in a sample may suggest a bottleneck in viral entry or stochasticity in viral replication.

\section{Transcriptome changes at single-cell resolution}

To understand transcriptional changes of the infected hAOs at a single-cell resolution, we employed two 10X Genomics single-cell RNA-seq experiments for uninfected and infected hAOs at $3 \mathrm{dpi}$ (to a throughput of $21.3 \mathrm{~Gb}$ and $28.5 \mathrm{~Gb}$, respectively). We captured 3,435 and 3,475 single-cells, respectively, with 15,703 UMls and 3,414 detectable genes per cell on average. Using a total of 6,910 single-cells, we performed unsupervised clustering using UMls of the human origin, and identified four distinct clusters (Fig. 4a): Cluster 1, mostly with uninfected alveolar cells (3,133 cells); Cluster 2, mostly with airway-like cells (361 cells; Supplementary Discussion); Cluster 3, alveolar cells with moderate levels of viral RNA transcripts (2,143 cells); and Cluster 4, cells disintegrating and likely close to cell death $(n=1,273)$. The cells in the experiment of infected hAOs were mostly distributed in Cluster 3 (2,142 cells; $61.6 \%$ of infected hAOs) and Cluster 4 ( 1,154 cells; $33.2 \%$ of infected hAOs) (Fig. 4b). Viral transcripts were found in the vast majority of infected hAO cells $(99.9 \% ; 3,471$ out of 
3,475 infected hAOs), validating our previous observation that most cells in the infected hAOs harbor viral transcripts (Fig. 2). The number of viral transcripts, however, was not uniformly distributed in all infected hAO cells, but enriched in Cluster 4 cells. Infected cells in Cluster 4 exhibited 13.7 times more viral UMI counts than cells in Cluster 3, on average (Fig. 4c; 1,904 vs. 139 UMls, respectively), despite cells in Cluster 4 containing relatively lower total UMI counts than ones in Cluster 3 (5,040 vs. 17,843 UMIs, respectively). When normalised with UMI counts for human genes, cells in Cluster 4 showed a $>30$ times higher viral RNA burden than cells in Cluster 3 (Fig 4d). Interestingly, the infected cells in Cluster 4 showed reduced expression of canonical hAT2 marker genes, including SFTPB (Surfactant Protein B) and NKX2-1 (NK2 homeobox 1) (Fig. 4e; Extended Data Fig. 6a). Compared with infected cells in Cluster 3, expression levels of ISGs, such as IFI44L and OAS3, were also highly reduced. Instead, these cells showed transcriptional induction of apoptosis mediator, GADD45B (growth arrest and DNA-damage-inducible, beta) and anti-apoptotic TNFAIP3 (tumor necrosis factor, alpha-induced protein 3), suggesting a catastrophic cellular pathway operating in a cell due to the extreme viral burdens.

Despite active protein expression (Fig. 1 f and 1g; Extended Data Fig. 1g), we found 14 cells $(0.4 \%)$ showing ACE2 transcripts, 579 cells (16.7\%) expressing TMPRSS2 transcripts, and 4 cells $(0.1 \%)$ coexpressing both in single-cell transcriptome sequencing (Fig. 4e). These proportions are low at face value, but are consistent with a previous observation ${ }^{41}$. Although the previous report also suggested that ACE2 RNA expression can be stimulated as an infection-mediated response, particularly in human airway cells, such a trend was not observed in our dataset.

\section{Effective number of viral entry for infection of an alveolar cell}

Finally, we statistically inferred the number of viral particles effectively entering each alveolar cell for infection. Although we incubated cells at an $\mathrm{MOI}$ of 1 on average, it is generally not known how many viral particles are necessary for effective infection of an alveolar cell. In an extreme scenario, one viral particle is sufficient. Alternatively, infection may be initiated with the entry of multiple viruses. We tracked the effective viral number of cellular entry using a mutation (NC_045512.2: 23,707C>U) as a viral barcode. From our sequencing, the mutation was estimated to be present at $4.3 \% \mathrm{VAF}$ in the initial viral pool for innoculation. If the first scenario dominantly applies, the infected alveolar cells will 
be dichotomized, i.e., $95.7 \%$ cells with solely wild-type virus and the rest of the cells with solely mutant virus, exclusively. Alternatively, if multiple viruses are effectively entering a cell, a large proportion of alveolar cells harbouring detectable mutant virus will have intermediate VAF for the mutation. In our single-cell transcriptomes of infected hAOs, 212 cells were informative with the viral mutation, containing at least two independent transcripts for the mutant locus. The majority of the cells harbouring the mutant allele did not have the wild-type allele present ( $n=8$ vs. 4 for cells showing solely mutant allele vs mixture of the quasispecies, respectively; Fig. 4f). In a more sophisticated statistical analysis, infection by single viral entry is estimated as $>2$ times more frequent than by multiple viral entry (69\% vs. $31 \%$, respectively; Fig. $\mathbf{4 g})$. Our calculation indicates that a single viral particle is mainly responsible for SARS-CoV-2 infection in most alveolar cells, although multiple viral entry is also possible. It may also reflect the viral interference in SARS-CoV-2 alveolar infection.

\section{Discussion}

In this study, we established conditions for optimised 3D long-term cultures of adult hAT2 cells, which provided an essential tool for studying initial intrinsic responses of SARS-CoV-2 infection. Single hAT2 cells were capable of self-constructing alveolus-like structures consisting of AT2 cell and differentiated AT1 cells. Mature hAT2 cells were maintained $>10$ months over multiple passages although self-renewal capacity and growth rate was reduced after 6 month in cultures. hAT1 cells were also lost from later culture, likely due to the persistent exposure to high WNT conditions allowing expansion of hAT2 cells over differentiation. Alteration of WNT activity in culture media (differentiation media) may enable to induce further AT1 cell differentiation.

hAOs showed remarkable phenotypic changes in the first few days after SARS-CoV-2 innoculation. Our single-cell transcriptome profiling identified two clusters of infected cells (Clusters 3 and 4), suggesting a distinct switch between cell states occurring during infection. If in vivo SARS-CoV-2 infection transforms hAT2 cells into Cluster 4-like cells showing the loss of hAT2 identity, the integrity and function of alveoli will be highly compromised. The route towards the quantum cellular change is of significant interest to be answered in future studies. To this end, we need more single-cell RNA sequencing from infected cells at earlier time points in combination with comprehensive highresolution imaging. 
The interferon response is the first line of host antiviral defense ${ }^{40}$. Contrary to a recent report ${ }^{42}$, we observed substantial ISGs in the alveolar cells induced by endogenously produced type I and III interferons. However, the induction of interferon responses was seen at 3 dpi in hAO models, 1-2 days later than the timing of viral amplification at $1 \mathrm{dpi}$. The timing of ISG induction may be earlier in vivo in concert with exogenous interferons from immune cells. For more physiological understanding, co-culturing SARS-CoV-2 infected hAO models with immune cells obtained from the same donor will be helpful.

In summary, our study highlights the power of feeder-free hAOs to elucidate the intrinsic responses of tissue damage including virus infection. Our data, including high-resolution electron microscopic images and the list of gene expression changes following infection, will be a great resource for the biomedical community to provide a deeper characterisation of SARS-CoV-2 infection specifically within adult hAT2 cells. We believe that our hAO models will enable more accurate and sophisticated analyses in the very near future, especially for studying the response of viral infection within vulnerable groups such as aged or diseased lungs, providing the opportunity to elucidate individual patient responses to viral infection. Furthermore, our models can be applied to other techniques, such as co-culture experiments with immune cells and robust in vitro screening of antiviral agents applicable to alveolar cells, in addition to being applicable for the study of the basic biology of alveolar cells as well as chronic disorders of the lung. 


\section{Methods}

\section{Human Tissues}

For the establishment of human lung organoid models, human distal lung parenchymal tissues from deidentified lungs not required for transplantation were obtained from adult donors with no background lung pathologies from Papworth Hospital Research Tissue Bank (T02233), and Addenbrookes Hospital (Cambridge University NHS foundations trust) under the collaboration of Cambridge Biorepository for Translational Medicine (CBTM) project. Appropriate Human Tissue Act (HTA) guidance was followed; For organoids used for viral infection and following analysis, human lung tissue was obtained from patients undergoing lobectomy surgery at Seoul National University Hospital (SNUH) with written informed consent from approval of the ethical committee (approval no. C-1809-137-975). Organoids for infection studies were established from adjacent normal tissues in lung cancer patients or an idiopathic pulmonary fibrosis (IPF) patient.

\section{Virus particle preparation for infection}

SARS-CoV-2 viral particles known as BetaCov/Korea/KCDC03/20206, ${ }^{31}$ were used for the infection study. The patient (KCDC03) was diagnosed with COVID-19 on January 26, 2020, after traveling to Wuhan, China. The viral particles were prepared from Vero cells infected with MOI 0.01 and grown under DMEM (Sigma) with 2\% FBS(Gibco), 1\% P/S(Gibco) for 48 hours at $37^{\circ} \mathrm{C} 5 \%$ CO2. Media was centrifuged with $2500 \mathrm{rpm}$ for $25 \mathrm{~min}$, and supernatant without cell debris was stocked at $-80^{\circ} \mathrm{C}$ with $4 \mathrm{x}$ $10^{6} \mathrm{pfu} / \mathrm{ml}$.

\section{Human lung tissue dissociation and flow cytometry}

Distal lung parenchymal tissue was processed as soon as possible in order to minimize cell yield loss and maintain cell viability. Briefly, fresh tissue was washed in cold PBS and minced into small $(1 \mathrm{~mm})$ pieces with a scalpel, followed by further dissociation using pre-warmed digestion buffer containing 2U/ mL Dispase II (Sigma, Corning), $1 \mathrm{mg} / \mathrm{mL}$ Dispase/Collagenase (Sigma) and $0.1 \mathrm{mg} / \mathrm{mL}$ DNase I (Sigma) in PBS at $37^{\circ} \mathrm{C}$ for $1 \mathrm{hr}$ with agitation. Tissue cell suspensions were filtered through a 100 $\mathrm{mM}$ cell strainer into a $50 \mathrm{~mL}$ falcon tube to remove cell debris, and washed with $10 \mathrm{~mL}$ of DMEM 
(Gibco, Thermofisher). Cells were centrifuged at $350 \mathrm{~g}$ for $10 \mathrm{~min}$, supernatant carefully aspirated, and cell pellet resuspended in $5 \mathrm{~mL}$ RBC lysis buffer for $5 \mathrm{~min}$ at room temperature (RT). The reaction was quenched using $5 \mathrm{~mL}$ of DMEM, and the entire $10 \mathrm{~mL}$ of cell suspension was transferred to a 15 $\mathrm{mL}$ falcon tube, followed by $10 \mathrm{~min}$ centrifugation at $350 \mathrm{~g}$. Supernatant was removed, and the cell pellet was resuspended in 10\% FBS in PBS (PF10 buffer) for counting. Cells were prepared for flow cytometry with primary antibodies CD31-APC (Biolegend, 303116), CD45-APC (Biolegend, 368512), EpCAM-FITC (Biolegend, 324204) and HTII-280-IgM (Terrace Biotech, TB-27AHT2-280) at 1:40 per 4 million cells for 30 min on ice. Following two washes with cold PF10 buffer and centrifugation at 350 $\mathrm{g}$ for $5 \mathrm{~min}$, cells were stained with secondary PE goat anti-mouse IgM (eBioscience, 12-5790-81) for HTII-280 at 1:100. Stained cells were washed with PF10 buffer, and counted using a hemocytometer to assess dilution required for final volume. Cells were diluted at a concentration of 30 million cells/ $\mathrm{mL}$ and filtered through a $35 \mu \mathrm{M}$ cell strainer into polypropylene FACS tubes. Cell sorting was performed on an Aria III fusion (BD Biosciences) using a $100 \mu \mathrm{M}$ nozzle, and data were analysed with FlowJo software (Tree Star, Inc.).

\section{In vitro organoid culture and passage}

Freshly isolated HTII-280+ and HTII-280-cells derived from CD31-CD45-EpCAM+ cells of human distal lungs were resuspended in Advanced DMEM/F12 (Thermofisher) base medium supplemented with $10 \mathrm{mM}$ Hepes (Gibco), $1 \mathrm{U} / \mathrm{mL}$ Penicillin/Streptomycin (Gibco), $1 \mathrm{mM} \mathrm{N}$-Acetylcysteine (Sigma), and $10 \mathrm{mM}$ Nicotinamide (Sigma). Growth factor-reduced (GFR-) Matrigel (Corning) was added to the cell suspension at a ratio of $1: 1$, and $100 \mu \mathrm{L}$ of suspension was added to a 24 -well transwell insert with a $0.4 \mu \mathrm{M}$ pore (Corning) so that there were approximately $10 \times 10^{3}$ cells per insert. GFR-Matrigel was allowed to solidify for $1 \mathrm{hr}$ at $37^{\circ} \mathrm{C}$, after which $500 \mu \mathrm{L}$ of pre-warmed alveolar media (base media supplemented with 1 x B27 (ThermoFisher), 10\% R-SPONDIN-1 (Cambridge Stem Cell Institute tissue culture core facility, manually produced), $50 \mathrm{ng} / \mathrm{ml}$ human EGF (Peprotech), $100 \mathrm{ng} / \mathrm{ml}$ human FGF7/KGF (Peprotech), 100 ng/ml human FGF10 (Peprotech), 100 ng/ml NOGGIN (Peprotech), 10 M SB431542 (Tocris) and $3 \mu \mathrm{M}$ CHIR99021 (Tocris)) was added to each lower chamber. For assessment of the effect of Wnt activity on hAT2 culture ability, primary cultures were also established without $\mathrm{CHIR99021.} \mathrm{Cultures} \mathrm{were} \mathrm{maintained} \mathrm{under} \mathrm{standard} \mathrm{cell} \mathrm{culture} \mathrm{conditions}\left(37^{\circ} \mathrm{C}, 5 \%\right.$ $\mathrm{CO}_{2}$ ), with media changes every 2-3 days. Y-27632 (10 $\mu \mathrm{M}$, Sigma) was added for the first $48 \mathrm{hr}$ of 
culture to promote cell survival. To avoid the growth of fungal and bacterial infection, $250 \mathrm{ng} / \mathrm{mL}$ Amphotericin B and $50 \mu \mathrm{g} / \mathrm{mL}$ gentamicin were added to culture medium for 5 days. For culture in 48 well plates, $5 \times 10^{3}$ cells were resuspended in $100 \%$ GFR-Matrigel, and allowed to solidify in a $20 \mu \mathrm{L}$ droplet per well at $37^{\circ} \mathrm{C}$ for $20 \mathrm{~min}$, followed by submersion in $250 \mu \mathrm{L}$ of pre-warmed medium. Organoids larger than $45 \mu \mathrm{M}$ in size were counted at day 14 of culture to assess organoid forming efficiencies, and were either fixed and stained at day 21 for analysis, or enzymatically dissociated into single cells for further culture without sorting. Organoid lines were passaged at different days depending on size, with culture days varying from 21-35 days. For passaging, Matrigel was disrupted by incubation with Dispase (Sigma) at $37^{\circ} \mathrm{C}$ for $45 \mathrm{~min}$, followed by single cell-dissociation through addition of trypLE (Gibco) for 5 min at $37^{\circ} \mathrm{C}$. The reaction was quenched with base medium, and cells were centrifuged at $350 \mathrm{xg}$ for $5 \mathrm{~min}$. Cells were resuspended in fresh GFR-Matrigel at a ratio of $5 \mathrm{x}$ $10^{3}$ (48-well plates) or $10 \times 10^{3}$ (24-well transwell inserts) cells as before. HTII-280- cells were cultured as with $\mathrm{HTII}-280^{+}$, although with a few minor differences. Bronchial organoids (hBOs) were passaged every 21-28 days due to accelerated growth compared with alveolar organoids (hAOs), and were cultured in previously reported medium conditions ${ }^{30}$ with the following concentration/factor edits; $100 \mathrm{ng} / \mathrm{ml}$ human FGF10, 10\% R-SPONDIN-1, 10 M SB431542 (instead of A83-01).

\section{Virus infection to organoids and Vero cells}

For $\mathrm{hAO}$ and $\mathrm{hBO}$, cells were recovered from Matrigel with a Recovery solution (Corning). Organoids were sheared with $1000 \mathrm{p}$ pipette tips or incubated with Accutase (Stem cell technology) at $37^{\circ} \mathrm{C}$ for 5 minutes. Organoids were resuspended with each organoid's media and were infected with virus multiplicity of infection (MOI) of 1 for 2 hrs at $37^{\circ} \mathrm{C} 5 \% \mathrm{CO}_{2}$. After virus infection, organoids were washed twice with Advanced DMEM/F12 with $1 \mathrm{U} / \mathrm{ml}$ Penicillin/Streptomycin, $10 \mathrm{mM} \mathrm{Hepes,} \mathrm{and} \mathrm{1 \%}$ Glutamax (v/v) (hereafter referred to as $\left.\mathrm{ADF}_{+++}\right)$and embedded with $50 \mu$ l of GFR-Matrigel (Corning) in 24-well plate (TPP). At least each well contained 10,000 cells. Media and cells resuspended in ADF+++ with Matrigel were taken at indicated time points. For viral particle release inside cells, cells were lysed by freezing at $-80^{\circ} \mathrm{C}$ and thawing. Live virus titers were determined by plaque assay and viral RNA titer was calculated using qPCR. For Vero cell infection, a virus with MOI 1 was poured on Vero cells directly, and cells with viruses were incubated for $1 \mathrm{hr}$ at $37^{\circ} \mathrm{C} 5 \% \mathrm{CO}_{2}$. After infection, infection media was removed and cells were incubated in normal media in $37^{\circ} \mathrm{C} 5 \% \mathrm{CO}_{2}$ condition. All 
work was performed in a Class II Biosafety Cabinet under BSL-3 conditions at Korea Center for Disease Control (KCDC).

\section{Immunofluorescence staining of paraffin-embedded organoids}

Organoids were fixed and embedded in a paraffin block ${ }^{43}$. Pre-cut $7 \mu \mathrm{M}$ paraffin sections were dewaxed and rehydrated (sequential immersion in xylene, 100\% EtOH, 90\% EtOH, 75\% EtOH, distilled water) and either stained with hematoxylin and eosin (H\&E) or immunostained. For antigen retrieval, slides were submerged into pre-heated citrate antigen retrieval buffer ( $10 \mathrm{mM}$ sodium citrate, $\mathrm{pH}$ 6.0) and allowed to boil for $15 \mathrm{~min}$. Slides were cooled in a buffer for $20 \mathrm{~min}$, washed in running water for 3 min, and permeabilised with $0.3 \%$ Triton-X in PBS for 15 min. Following permeabilisation, organoid sections were blocked for $1 \mathrm{hr}$ in $5 \%$ normal donkey serum in PBS at RT, and incubated with primary antibody mixes overnight at $4{ }^{\circ} \mathrm{C}$ at the following dilutions; rabbit pro-SFTPC $(1: 500$, Millipore, Ab3786), mouse anti-HTII-280 (1:500, Terrace Biotech, TB-27AHT2-280), rat anti-SCGB1A1 (1:200, R\&D systems, MAB4218), rabbit anti-KRT5 (1:500, Biolegend, 905501), mouse anti-ABCA3 (1:300, Seven Hills Bioreagents, WRAB-ABCA3), and mouse anti-TP63 (1:500, Abcam, ab735), rabbit antiHOPX (1:200, Santa Cruz Biotechnologies, sc-30216), and sheep anti-PDPN (1:200, R\&D, AF3670). Antibodies were removed with three PBS washes, and samples were incubated with Alexa Fluorcouple secondary antibodies (1:1000, Jackson Laboratory) for $1 \mathrm{hr}$ at RT. Following PBS washes, nuclei were stained with DAPI for 5 min, slides mounted with Rapiclear (Sunjin lab), and sealed with clear nail polish. For Lysotracker ${ }^{\mathrm{TM}}$ staining of lysosomes, live organoids in 48-well plates were incubated in situ with $50 \mathrm{ng} / \mu \mathrm{L}$ of Lysotracker ${ }^{\mathrm{TM}}$ (Invitrogen, L12492), diluted in pre-warmed expansion medium, for $1 \mathrm{hr}$ at $37^{\circ} \mathrm{C}$. Lysotracker was removed, and organoid/matrigel suspension was carefully washed for 5 min in PBS, followed by addition of fresh, pre-warmed expansion medium. Cells were protected from light and imaged immediately using an EVOS cell imaging system.

\section{Immunofluorescence staining of infected organoid with cryosection}

Organoids were fixed in $4 \%$ paraformaldehyde (PFA) for $3 \mathrm{hrs}$ at ice, and then dehydrated in PBS with $30 \%$ sucrose (v/v) (Sigma). Organoids were embedded with optimal cutting temperature (OCT) compound (Leica) and cut with $10 \mu \mathrm{M}$. Organoid section was blocked with 5\% normal donkey serum in PBS $1 \%$ triton-X (Sigma). Sections were incubated with primary antibodies overnight at $4{ }^{\circ} \mathrm{C}$, 
washed three times with PBS. Organoids were incubated host matched Alexa Fluor-couple secondary antibodies (Jackson Laboratory) with PBS for $1.5 \mathrm{hr}$ at RT. Following DAPI incubation, slides were mounted. Antibodies were E-cadherin (R\&D, AF748, 1:300), NP (Sino, 40143-MM05, 1:200), Spike S1 protein (Sino, 10030005, 1:200), ACE2 (abcam, ab15348, 1:400), TMPRSS2 (abcam, EPR3861,1:400)

\section{Transmission electron microscopy}

Human alveolar organoids were fixed with $2.5 \%$ glutaraldehyde in $0.1 \mathrm{M} \mathrm{PBS}$ overnight at $4{ }^{\circ} \mathrm{C} 44$. Organoids were washed with PBS and post-fixed with $2 \%$ osmium tetroxide for $1.5 \mathrm{hr}$. The fixed sample was dehydrated in graded ethanol, substituted with propylene oxide, and finally embedded in EMbed-812 resin (EMS). Polymerization was performed at $60^{\circ} \mathrm{C}$ for $24 \mathrm{hrs}$. Ultrathin (100 nm) sections were prepared using an ultramicrotome (Leica, EM UC7). Images were captured with a transmission electron microscope (FEI Tecnai G2 spirit TWIN, eagle 4K CCD camera) at 120kV acceleration voltage. All work was carried out in the EM \& Histology Core Facility, at the BioMedical Research Center, KAIST.

\section{RNA purification}

For viral RNA extraction, cells were resuspended with Matrigel with media and frozen at $-80^{\circ} \mathrm{C}$ and then thawed. Thawed solution was processed through the QIAamp Viral RNA Mini Kit. For mRNA extraction, cells were recovered from Matrigel by a Recovery solution (Corning) and then centrifuged at $300 \mathrm{~g}$ for $5 \mathrm{~min}$ at $4{ }^{\circ} \mathrm{C}$. Cell pellets were lysed, and RNA was extracted through RNeasy Plus Mini Kit. For viral RNA extraction from media, $140 \mu \mathrm{l}$ of media was processed through QIAamp Viral RNA Mini Kit.

\section{Assessment of lung lineage transcripts by qRT-PCR}

Freshly sorted HTII-280+ and HTII-280- cells were lysed with TRIzol, and RNA was extracted. RNA was reverse transcribed using SuperScript IV (Thermo Fisher Scientific), and were assessed using the following Taqman probes; SFTPC (Hs00951326_g1), TP63 (Hs01114115_m1), SCGB1A1 (Hs00171092_m1). 


\section{Viral RNA copy number calculation with qRT-PCR}

Viral RNA samples were reverse-transcribed using SuperScript IV (Thermo Fisher Scientific). Viral N3 gene was targeted for qRT-PCR. Nucleotide sequences of the probes as below (CDC).

\section{9-nCoV_N3-F: 5' GGGAGCCTTGAATACACCAAAA 3'}

2019-nCoV_N3-R: 5' TGTAGCACGATTGCAGCATTG 3'

Samples were prepared triplicate from solution. Viral RNA copy number was calculated by comparing the standard curve of virus N3 gene. For generating the standard curve, the positive viral RNA template was amplified with CDC designed N3 gene primers and cloned into pGEM-T Easy vector (Promega, USA). The resultant plasmid DNA was linearized with Pstl restriction enzyme and purified with a QIAquick PCR Purification Kit (QIAGEN, Germany). Purified template was in vitro transcribed by RiboMAX ${ }^{\mathrm{TM}}$ Large Scale RNA Production System with T7 RNA polymerase (Promega, USA). RNA transcript was further purified with the NuceloSpin RNA Mini kit (MACHEREY-NAGEL, Germany) and quantified with spectrophotometer at $260 \mathrm{~nm}$. In vitro transcribed RNA was serially diluted and reverse transcribed for the quantitative real time $\mathrm{PCR}^{45}$.

\section{Live virus titer calculation with plaque assay}

Matrigel was sheared with the organoid media and frozen at $-80^{\circ} \mathrm{C}$ once. Thaw the solution and dilute by scale of 10 . Each well containing Vero cells in 12 wells were infected with the diluted solution respectively at $37^{\circ} \mathrm{C}, 5 \% \mathrm{CO}_{2}$ for $1 \mathrm{hr}$. After infection, remove infection media and wash the Vero cells with PBS two times, mixed agar and Modified Eagle's Medium (Thermofisher) were poured on each well. When agar mixture was hardened, fix each well with 4\% PFA for 3 days, and stain with crystal violet (Sigma). When there are individual spots, the original solution's viral titer was calculated.

\section{Bulk RNA sequencing and data processing}

Extracted cellular RNA was processed through Truseq Stranded Total RNA Gold kit, and cDNA library was sequenced $2 \times 100$ bp using Hiseq 2500. Fastq file was aligned to GRCh38 with virus sequence (NC 045512.2 from NCBI) using STAR ${ }^{46}$ and normalized RNA expression was calculated using RSEM ${ }^{47}$. Differentially expressed genes are found from DEseq2 ${ }^{48}$. We obtained enriched gene sets 
using in-house scripts. For mutation calling, we used Strelka2 ${ }^{49}$, Varscan $2^{50}$, and Samtools ${ }^{51}$, and then manually checked the position through IGV52.

\section{Single-cell RNA sequencing and data processing}

Fastq file was aligned and each UMI count was calculated using Cell Ranger software provided by the manufacturer (10X Genomics). Cells with mitochondria RNA percent $<25 \%$, total RNA number $>200$ subsets were used for downstream analysis.

Starting from the 10X gene counts, we have normalized data as follows. The 10X data includes SARS-CoV-2 genome as an extra gene besides 19,941 human genes. For uninfected cells, such SARS-CoV-2 gene would have zero read count, while for infected cells, the gene could account for a large portion of total reads. One of the goals of normalisation is to remove technical difference such as sequencing coverage before comparing gene expression levels. As our interest is to compare expression levels between uninfected and infected cells on human genes, we have applied a normalisation method ('scater R package's 'logNormCounts' function) to human genes as a whole set. The method calculated a scaling factor for each cell based on total human gene count, then scaled all genes before taking log-transformation. Using the same scaling factor learned during human gene normalisation, SARS-CoV-2 count was also normalized. For clustering, we combined single-cell data from infected and uninfected hAOs. Unsupervised clustering was performed using a shared nearest neighbor (SNN) based clustering algorithm in Seurat ${ }^{53}$. Contaminated cells $(<3 \%)$ were discarded. In house R scripts were used for more downstream analyses.

\section{Statistical inference on the effective number of viral entry}

To assess whether alveolar cells tend to be infected by a single viral particle or multiple particles, we employed a likelihood approach. As a proof-of-concept, we assumed only two scenarios exist, one supporting a single viral entry and the other supporting double viral entry, then aimed to estimate the proportion of cells with a single viral particle $(w)$. Out data consists of the observed reference $\left(n_{\text {cell }}^{r}\right)$ and variant $\left(n_{\text {cell }}^{v}\right)$ read counts for each of the 547 reporting at least one read at the mutation site of NC_045512.2:23,707. Assuming a sequencing error rate $(\varepsilon)$ of $0.1 \%$, which will cover any Illumina sequencing errors or misalignment, the likelihood of data given the weight supporting a single virus scenario $(w)$ was computed as follows. 


$$
\begin{aligned}
& L_{\text {cell }}(w)=w \times P\left(\text { Data }_{\text {cell }} \mid \text { single entry }\right)+(1-w) \times P\left(\text { Data }_{\text {cell }} \mid \text { double entry }\right) \\
& P\left(\text { Data }_{\text {cell }} \mid \text { single entry }\right)=P(G=R) \times P\left(n_{\text {cell }}^{r}, n_{\text {cell }}^{v} \mid \text { va } f_{\text {cell }}=\varepsilon\right)+ \\
& P(G=V) \times P\left(n_{\text {cell }}^{r}, n_{\text {cell }}^{v} \mid v a f_{\text {cell }}=1-\varepsilon\right) \\
& P\left(\text { Data }_{\text {cell }} \mid \text { double entry }\right)=P(G=R R) \times P\left(n_{\text {cell }}^{r}, n_{\text {cell }}^{v} \mid v a f_{\text {cell }}=\varepsilon^{2}\right)+ \\
& P(G=R V) \times P\left(n_{\text {cell }}^{r}, n_{\text {cell }}^{v} \mid v a f_{\text {cell }}=0.5\right)+ \\
& P(G=V V) \times P\left(n_{\text {cell }}^{r}, n_{\text {cell }}^{v} \mid v a f_{\text {cell }}=(1-\varepsilon)^{2}\right)
\end{aligned}
$$

$G$ stands for the viral genotype within each cell, and $R$ and $V$ denote for the wildtype and mutant alleles in the genotype, respectively. $v a f_{\text {cell }}$ refers to the true mutant allele frequency within an infected cell given the viral genotype after accounting for the sequencing error rate.

For both single- or double-entry scenarios, the prior for each viral genotype was computed using the mutant allele frequency observed from the whole cell population $\left(v a f_{\text {pop. }} ; 4.3 \%=42\right.$ variant reads out of 986 reads). For example, the prior of having the genotype with two mutant alleles in a double-entry scenario is computed as follows:

$$
P(G=V V)=v_{\text {cell }}^{2}
$$

Within a cell, the probability of observing the number of reference and variant reads given the mutant allele frequency, $P\left(n_{\text {cell }}^{r}, n_{\text {cell }}^{v} \mid v a f_{\text {cell }}\right)$, was computed using binomial distribution.

\section{Supplementary discussion}

\section{Basal-like cells and hAT2 enrichment in single cell RNA sequencing}

We observed a distinct transcriptional feature of cells in Cluster 2 which express lower levels of canonical hAT2 marker genes, including SFTPC but detectable levels of airway marker genes, including SOX2, TP63, KRT5, and KRT17 (Extended Data Fig. 6b). These expression patterns were not affected 
bioRxiv preprint doi: https://doi.org/10.1101/2020.07.10.194498; this version posted July 10, 2020. The copyright holder for this preprint

(which was not certified by peer review) is the author/funder, who has granted bioRxiv a license to display the preprint in perpetuity. It is made available under aCC-BY-NC-ND 4.0 International license.

by virus infection. hAT2 cells expressing airway markers such as SOX2 were seen in chronic lung diseases such as lung cancer and idiopathic pulmonary fibrosis (IPF) 54,55 , representing pathologic phenotypes of alveolar bronchiolization. A recent study also suggested the potential transition of hAT2 cells to KRT5+ basal-like cells in the context of IPF27. Given the fact that the hAOs used for our singlecell RNA sequencing study were derived from hAT2 cells isolated from adjacent normal counterparts of lung cancer and/or IPF, it is likely that this transcriptional feature reflects the cellular status of original tissues rather than virus-associated phenotype. This finding suggests that our hAO models maintain the pathophysiologic features of original tissues although we used apparently normal background regions for our hAO establishments. Further long-term tracing of changes in cellular identities and states in response to virus infection in hAO cells will be of significant interest to understand the progression of pathologic features and reparative mechanisms for developing therapeutic interventions.

Furthermore, from our scRNAseq analysis, most captured cells were hAT2 cells. It is likely that this might result from the enrichment of hAT2 cells in our hAOs (P2) and the nature of fragile hAT1 cells during the procedure of single-cell preparation for scRNAseq. 


\section{Supplementary Tables}

Supplementary Table 1. RNA expression levels (TPM) of all genes in seven human alveolar organoid samples.

Supplementary Table 2. RNA expression levels (TPM) of all genes in seven human bronchial (airway) organoid samples.

Supplementary Table 3. Twenty single base substitutions of SARS-CoV-2 which were detected in infected organoids.

\section{Acknowledgements}

We thank Jinwook Choi (Wellcome-MRC Cambridge Stem Cell Institute), Yong Man Han, Eui-Cheol Shin, Heung Kyu Lee, Su-Hyung Park, Jeong Seok Lee, Ryul Kim, Myungsuk Choi (KAIST), Jung-Ki Yoon (Seoul National University Hospital), Yoon Ho Kim (Seoul National University Cancer Research Institute), and Jong-Yeon Shin (Macrogen Inc.) for valuable discussion, productive comments, and technical help; Irina Pshenichnaya (Histology), Peter Humphreys (Imaging), Simon McCallum (Flow cytometry, Cambridge NIHR BRC Cell Phenotyping Hub), and Cambridge Stem Cell Institute core facilities for technical assistance. This work was supported by Suh Kyungbae Foundation (SUHF18010082 to Y.S.J.); National Research Foundation of Korea (for Brain Pool Program NRF2019H1D3A2A02061168 to Y.S.J and S.Y.K; Leading Researcher Program NRF2020R1A3B2078973 to Y.S.J) and Institute for Basic Science (IBS-R025-D1, G.Y.K) funded by Ministry of Science and ICT of Korea. J.-H.L was supported by Wellcome and the Royal Society (107633/Z/15/Z) and European Research Council Starting Grant (679411). K.V.E was supported by the Biotechnology and Biological Sciences Research Council Industrial CASE (BBSRC iCASE) studentships (BB/R505328/1).

\section{Author contributions}

Conceptualization, Y.S.J. and J.-H.L.; hAO development, K.V.E., J.-H.L.; hAO establishments and characterisation, K.V.E., J.Y., T.K., J.-H.L.; Specimen preparation; K.J.N, K.M., K.S., Y.T.K., K.M., 
K.S.P.; Infection, J.K., J.Y., T.K., Y.-I.J., B.-S.C; Confocal microscope, K.V.E., S.P.H., G.Y.K.;

Electron microscope, Y.H., T.K., H.M.K.; Transcriptome analysis, J.Y., T.K., K.Y., S.Y.K., Y.S.J.;

Statistics, S.Y.K.; Writing, J.Y., T.K., K.V.E., T.B., S.-H.K., G.Y.K., Y.S.J., J.-H.L.; Manuscript

finalization, all authors; Supervision Y.S.J. and J.-H.L.

\section{Declaration of interests}

The authors declare no competing interests.

\section{Materials availability}

All unique organoids generated in this study are available from Young Seok Ju or Joo-Hyeon Lee with a completed Materials Transfer Agreement.

\section{Data availability}

Bulk RNA and single cell RNA sequencing datasets will be uploaded on the European Genome-

Phenome Archive (EGA). Accession ID is not assigned yet. 
bioRxiv preprint doi: https://doi.org/10.1101/2020 07 10.194498; this version posted July 10, 2020. The copyright holder for this preprin (which was not certified by peer review) is the author/funder, who has granted bioRxiv a license to display the preprint in perpetuity. It is made available under aCC-BY-NC-ND 4.0 International license.

Figures and legends

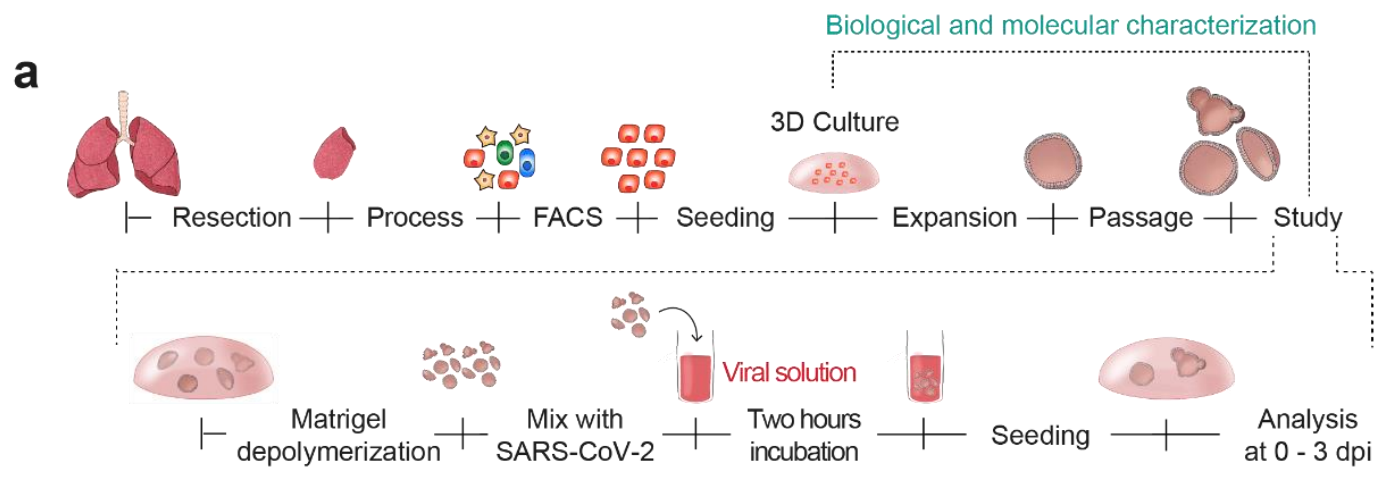

b

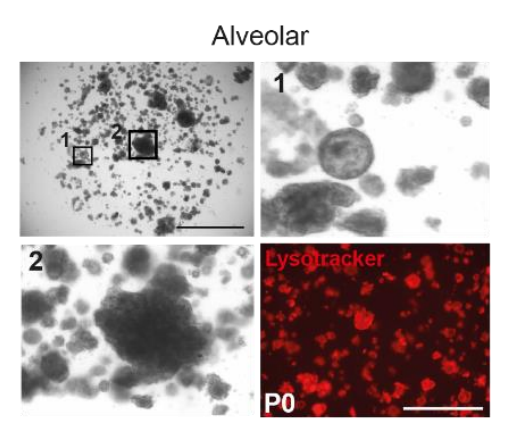

d

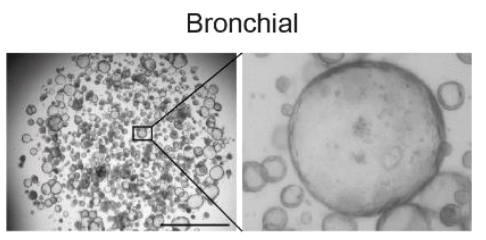

f

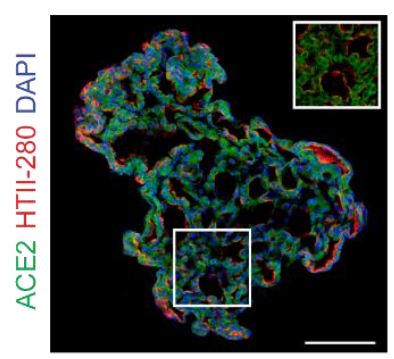

i

$\rightarrow$ Vero cell $\rightarrow$ hAO $\rightarrow$ hBO

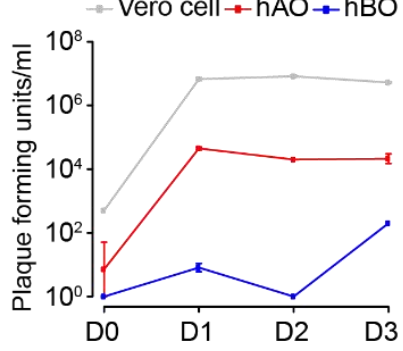

C

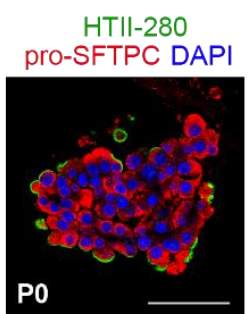

Alveolar

ABCA3 DAPI

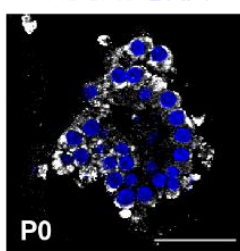

pro-SFTPC DAPI

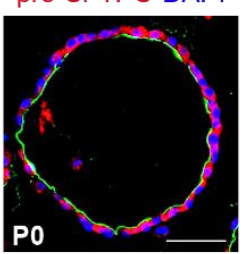

e
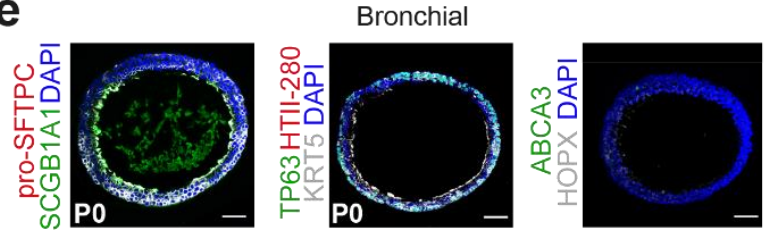

h

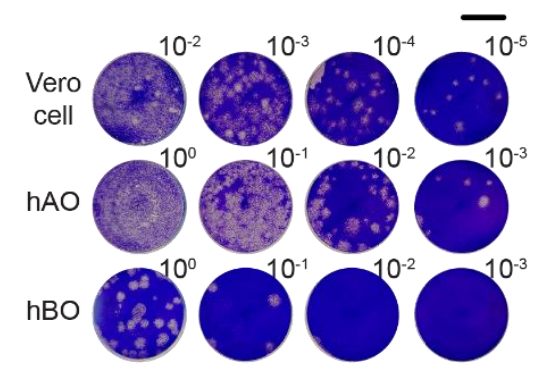

k

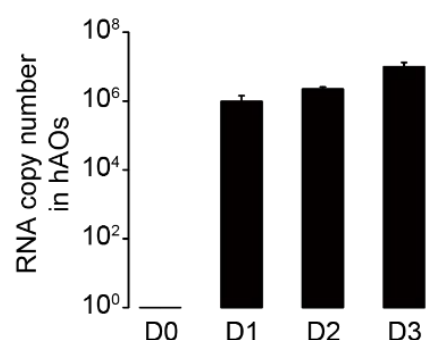

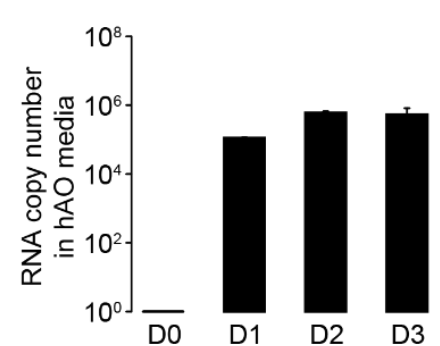

Figure 1. Establishment of long-term and three-dimensional human alveolar type 2 cell culture and SARS-CoV-2 infection in the three-dimensional model system 
a, Schematic diagram outlining the method for dissociation and processing of human adult lung parenchymal tissues for in vitro three-dimensional cultures (top). Schematic illustration of SARS-CoV2 infection experiments in this study (bottom). b, Representative bright-field images of hAOs derived from HTII-280+ hAT2 cells at day 28 in culture. Insets (top left) show high-power view of cystic-like (top right) and budding-like (bottom left) alveolar organoids. Scale bar, $2000 \mu \mathrm{m}$. Of note, the majority of hAOs exhibited uptake of Lysotracker (red), indicative of mature AT2 cells (bottom right). Scale bar, $1000 \mu \mathrm{m}$. c, Immunofluorescent staining of hAOs expressing AT2 markers. Left and right; HTII-280 (for hAT2, green), pro-SFTPC (for hAT2, red). Middle; ABCA3 (for hAT2, white). DAPI (blue). Scale bar, $50 \mu \mathrm{m}$. d, Representative bright-field images of hBOs derived from HTII-280- non-hAT2 cells at day 14 in culture. Insets (left) show high-power view (right). Scale bar, $2000 \mu \mathrm{m}$. e, Immunofluorescent staining of hBOs expressing airway lineage markers. Left; SCGB1A1 (for secretory, green), pro-SFTPC (for hAT2, red). Middle; KRT5 (basal, white), TP63 (basal, green), HTII280 (for hAT2, red). Right; ABCA3 (for hAT2, green), HOPX (for hAT1, white). DAPI (blue). Scale bar, $50 \mu \mathrm{m}$. f, Immunofluorescent staining of ACE2 (green) and HTII-280 (red) in hAOs. HTII-280 is stained in the apical membrane of hAT2 cells. Scale bar, $100 \mu \mathrm{m} . \mathbf{g}$, Immunofluorescent staining of TMPRSS2 (green) in hAOs. Scale bar, $100 \mu \mathrm{m}$. h, Representative images for plaque assay at $3 \mathrm{dpi}$. Dilution factors are shown in numbers on the right upper corner. Scale bar, $1 \mathrm{~cm}$. i, Plaque assay showing that SARS-CoV-2 actively replicates in hAOs at $1 \mathrm{dpi}$. SARS-CoV-2 can infect hBOs, but the viral amplification is much lower than in hAOs. Given the different culture and viral infection techniques between 2D Vero cells and 3D organoids, direct comparison is not applicable between Vero cells and hAOs. Error bars represent SEM. $n=2$. j, Quantitative PCR (qPCR) analysis for measuring the viral RNA levels in lysed hAOs. Error bars represent SEM. $n=3 . \mathbf{k}, q P C R$ analysis for measuring the viral RNA levels in hAO media. Error bars represent SEM. $n=3$. 
bioRxiv preprint doi: https://doi.org/10.1101/2020.07 10.194498; this version posted July 10, 2020. The copyright holder for this preprint (which was not certified by peer review) is the author/funder, who has granted bioRxiv a license to display the preprint in perpetuity. It is made available under aCC-BY-NC-ND 4.0 International license.

a
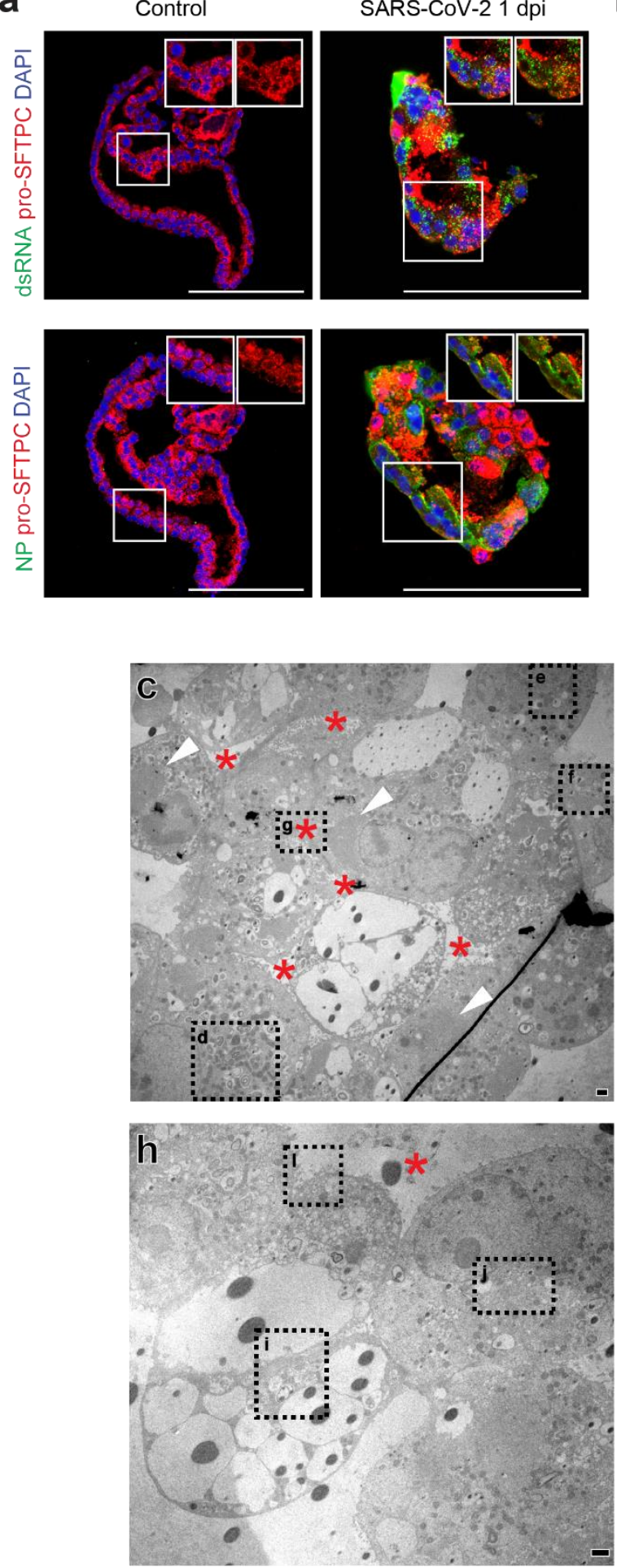

b

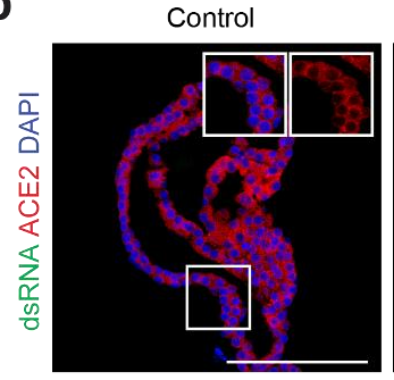

SARS-CoV-2 1 dpi
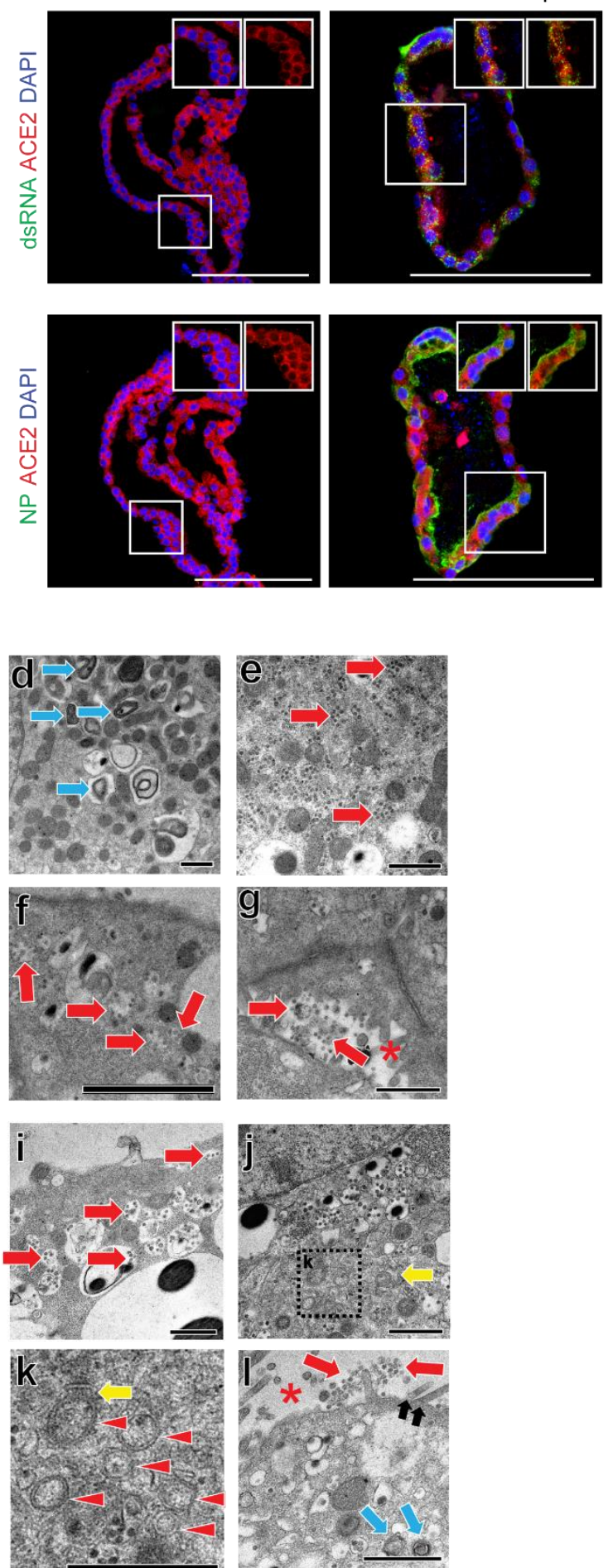

Figure 2. Confocal and transmission electron microscopic images of SARS-CoV-2 infected hAOs

a, SARS-CoV-2 infected hAOs at 1 dpi. Viral nucleocapsid protein (NP) and double-stranded RNA (dsRNA) are costained with pro-SFTPC. At 1 dpi, SARS-CoV-2 highly infects hAOs which show 
bioRxiv preprint doi: https://doi. org/10.1101/2020.07.10.194498; this version posted July 10, 2020. The copyright holder for this preprint

(which was not certified by peer review) is the author/funder, who has granted bioRxiv a license to display the preprint in perpetuity. It is made available under aCC-BY-NC-ND 4.0 International license.

punctuated pattern of pro-SFTPC (red). Scale bar, $50 \mu \mathrm{m}$. b, The viruses are identified by dsRNA (top right) and NP (bottom right). Infected hAOs express ACE2 (red). Viral dsRNA (green) appears punctuated. More than $80 \%$ of hAT2s are infected at 1 dpi. Scale bar, $50 \mu \mathrm{m}$. c, Transmission electron microscopic image of an entire hAO structure. Small alveolar spaces are shown between hAT2s (red asterisk). Aggregated viral particles are sometimes observed in electron-dense areas of the hAOs (white arrowhead). Scale bar (c-l), $1 \mu \mathrm{m}$. d, Lamellar bodies (skyblue arrow), consisting of pulmonary surfactants, are frequently observed in hAOs. e, Hundreds of SARS-CoV-2 particles in the cytosol of hAT2 cells (red arrow). f, Multiple viral particles in vesicular structures. g, SARS-CoV-2 viral particles secreted into an alveolar space of hAOs. $\mathbf{h}$, Another low magnification image of a hAO.

Some cells contain large pathologic vacuoles. i, Large vacuoles usually do not include virus particles, but small vesicles contain many virus particles. j, Viral containing vesicles, aggregated in the vicinity of zippered endoplasmic reticulum (ER; yellow arrow). $\mathbf{k}$, Double membrane vesicles (red arrowhead) located near zippered ER. I, Secreted viral particles in the apical lumen of hAOs. Microvilli (black arrow) and lamellar body (skyblue arrow) are shown at the apical side of hAT2s. 
a

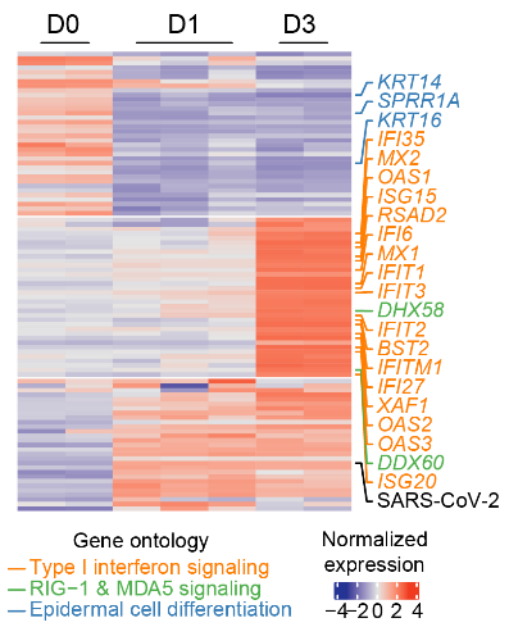

C

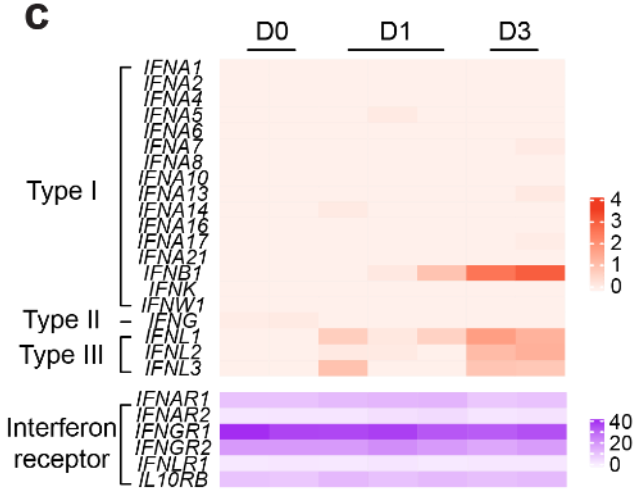

e
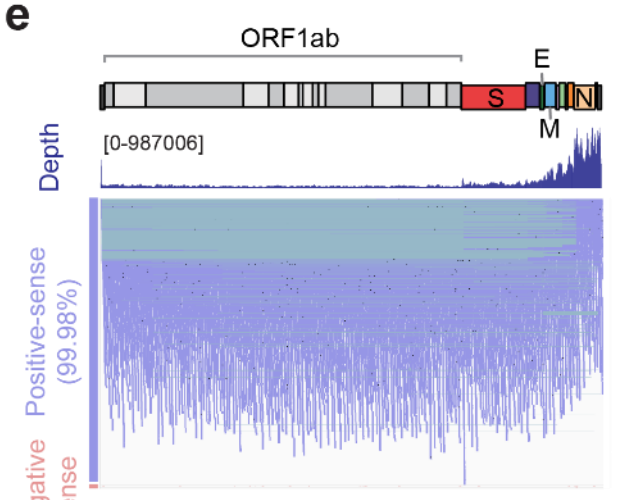

NC_045512.2:1-29,903

(No. of reads $=13,050,025$ b

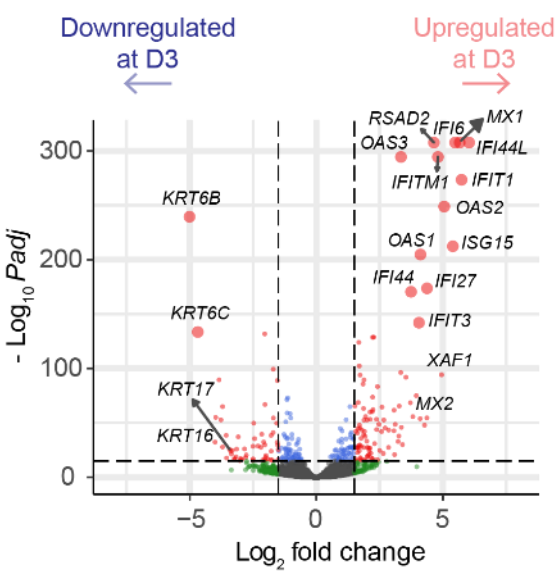

d
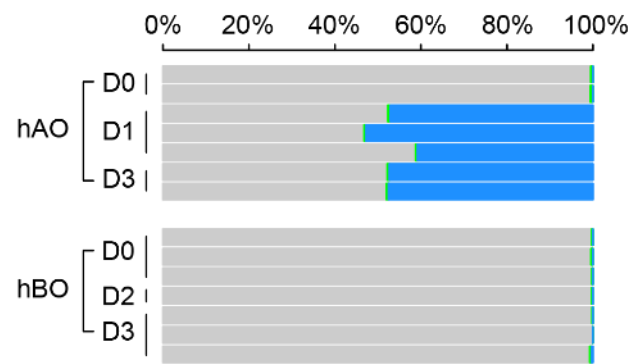

= Genome $=$ Mitochondria $=$ SARS-CoV-2

f

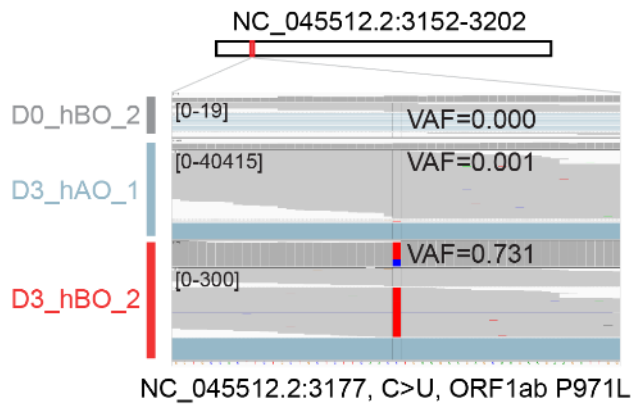

\section{Figure 3. RNA-seq analyses of infected hAOs and hBOs}

a, Heatmap of the most variable 100 genes among three groups of hAOs at 0, 1 and 3 dpi. Genes related to type I interferon signal pathway are highly elevated at $3 \mathrm{dpi}$, while SARS-CoV-2 expression reach plateau at $1 \mathrm{dpi}$. $\mathbf{b}, \mathrm{A}$ volcano plot showing differentially expressed genes between hAOs at 0 dpi and 3 dpi. Most highly upregulated genes are in type I interferon signaling pathway. c, Transcriptional changes of interferon genes in the infected hAOs. The rise of IFNB1, IFNL1, IFNL2, 
bioRxiv preprint doi: https://doi.org/10.1101/2020.07.10.194498; this version posted July 10, 2020. The copyright holder for this preprint

(which was not certified by peer review) is the author/funder, who has granted bioRxiv a license to display the preprint in perpetuity. It is made available under aCC-BY-NC-ND 4.0 International license.

and IFNL3 are observed in hAOs. d, Proportion of viral RNA reads in the hAO and hBO

transcriptomes. Compared to hAOs, the portion of viral reads is much lower in hBOs. e, Positivesense viral RNAs are dominant (99.98\%) over negative-sense viral RNAs. f, An example of missense mutation (NC_045512.2: 3,177C>U) detected from hBO transcriptome at 3 dpi. The variant allele fraction (VAF) of the substitution is 0.731 in an infected hBO sample, much higher than that in other infected cells. 

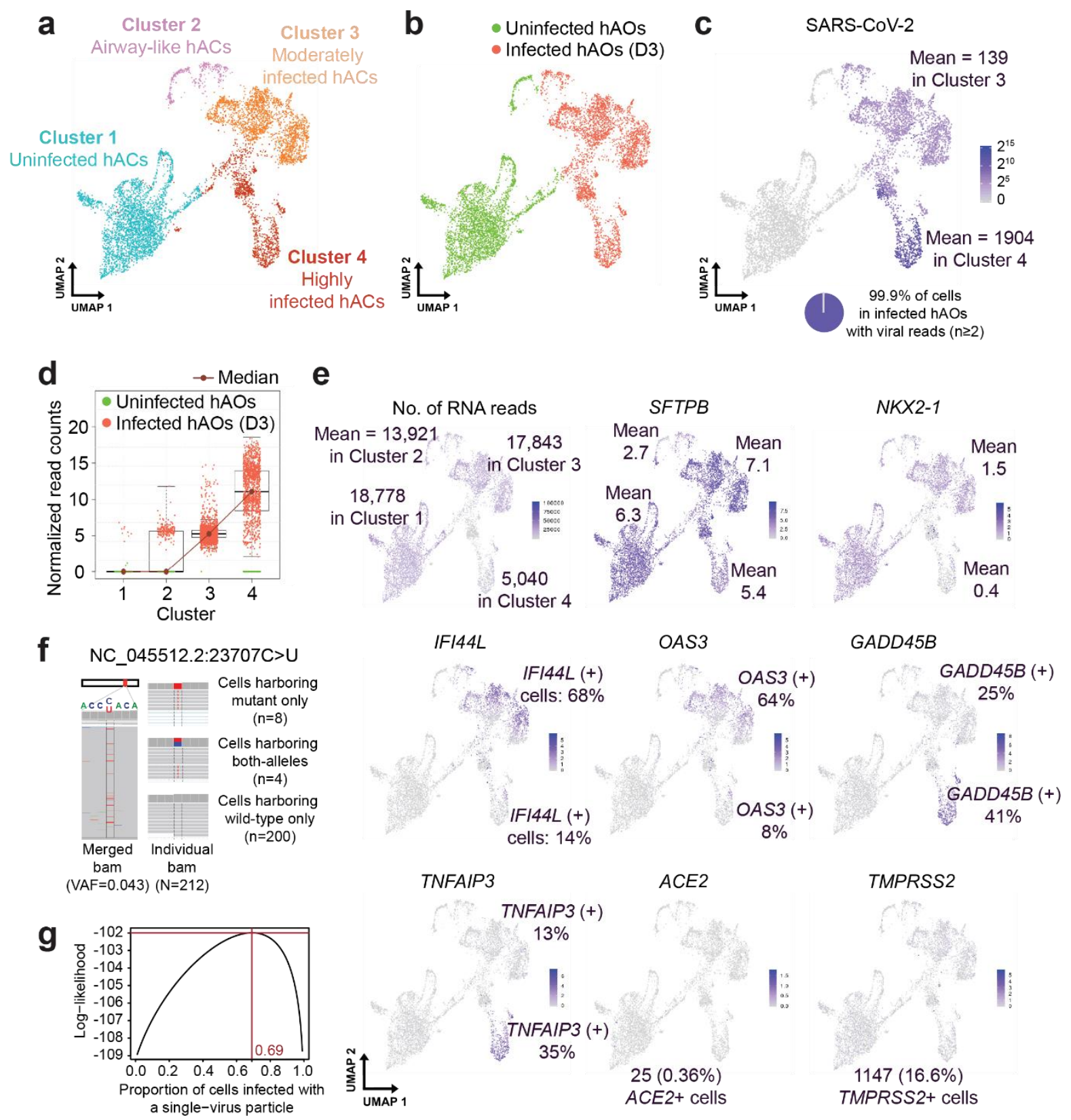

Figure 4. Single-cell transcriptome analysis of uninfected and infected hAOs.

a, Unsupervised clustering of uninfected and infected human alveolar cells ( $3 \mathrm{dpi}$ ) in the UMAP plot.

b, Experimental conditions of the dataset projected on the UMAP plot. c, Numbers of viral transcripts in each single-cell. Most cells harbor $\geq 2$ viral reads. $\mathbf{d}$, Normalized levels of SARS-CoV-2 viral read counts in each cluster. e, Expression of genes of interest in single-cells of each cluster. $\mathbf{f}$, Distribution of single base substitution (variant allele fraction $(\mathrm{VAF})=4.3 \%$ in original viral particles) in each single cell. g, Maximum likelihood estimation for the proportion of cells infected with a single virus. This 
bioRxiv preprint doi: https://doi.org/10.1101/2020.07.10.194498; this version posted July 10, 2020. The copyright holder for this preprint (which was not certified by peer review) is the author/funder, who has granted bioRxiv a license to display the preprint in perpetuity. It is made available under aCC-BY-NC-ND 4.0 International license.

estimation indicates that infection by a single virus is more common in hAT2s rather than by multiple viruses. 
a
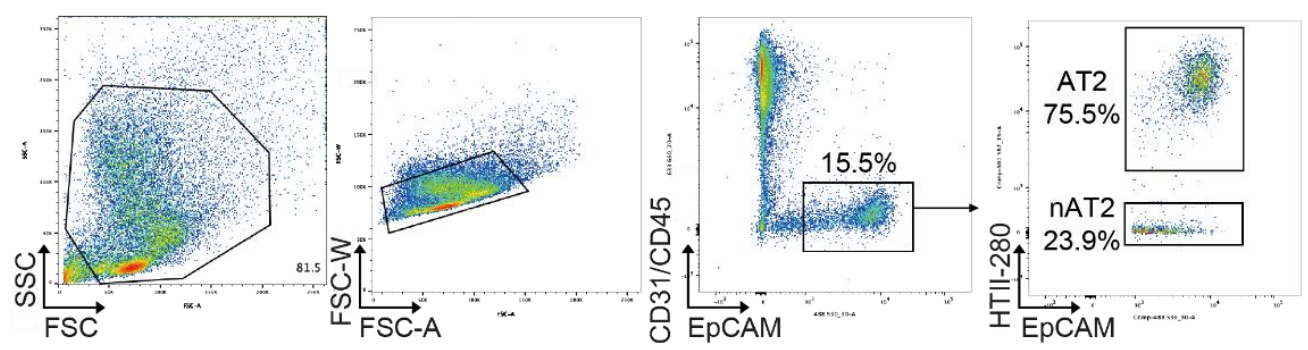

b

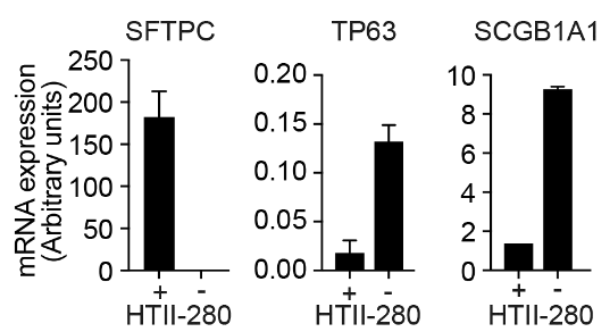

C

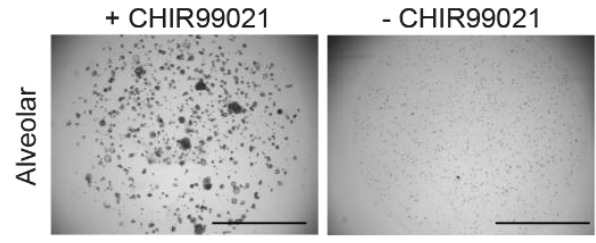

d
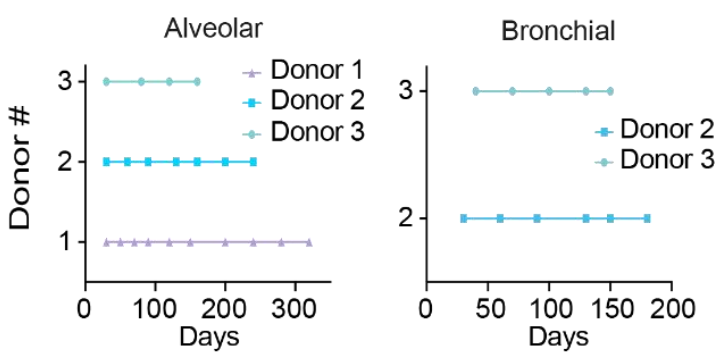

e
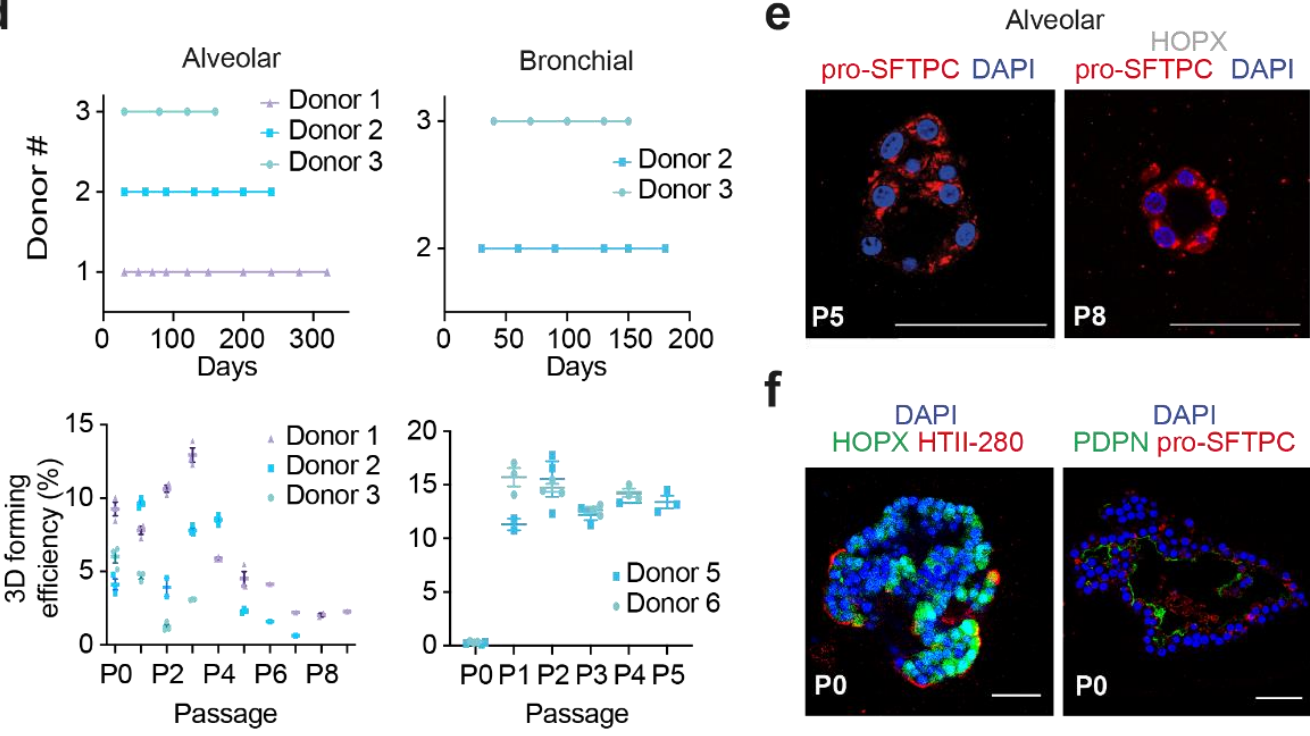

f
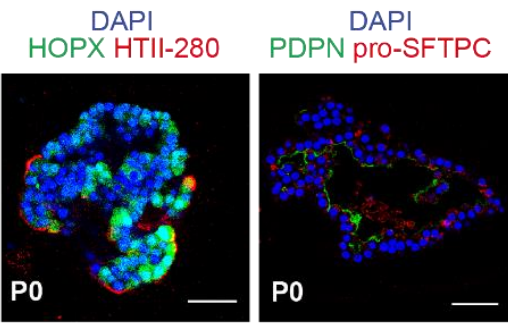

g
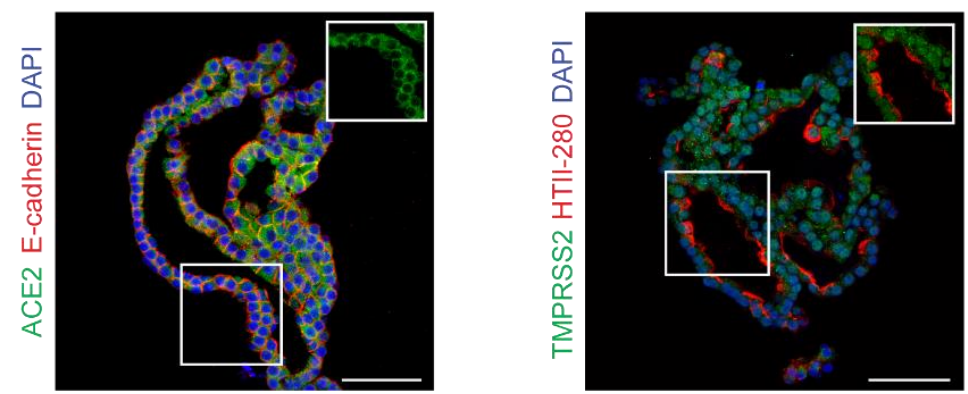

\section{Extended Data Figure 1. Establishment and characterisation of three-dimensional culture of}

\section{human alveolar type 2 cells}

a, Representative flow cytometry analysis for isolation of hAT2 cells (CD31-CD45-EPCAM+HTII-280 $)$

and non-hAT2 cells (nAT2, CD31-CD45-EPCAM+HTII-280-) from human distal lung parenchymal 
tissues. b, Quantitative PCR (qPCR) analysis of isolated primary human epithelial lung cells in (a) for lung lineage marker genes revealed that $\mathrm{HTII}-280^{+}$cells expressed much higher level of the AT2 cell marker SFTPC, while HTII-280 cells expressed virtually no SFTPC and instead expressed higher levels of the airway markers TP63 and SCGB1A1. Data is the mean \pm SEM of two technical replicates, and is expressed as arbitrary mRNA expression. c, Representative bright-field images of organoids derived from hAT2s in complete medium (left) and withdrawal of CHIR99021 (right). Scale bar, $2000 \mu \mathrm{m}$. d, Top; Organoid cultures for alveolar or bronchial cells from different donors were passaged at various time points depending on growth. Each point represents a single passage. Bottom; Statistical quantification of organoid forming efficiency at day 14-28 up to 9 passages (alveolar) and 5 passages (bronchial). Each individual dot represents one technical replicate, and data are presented as mean \pm SEM for each individual donor sample. $\mathrm{N}=3$ for $\mathrm{hAOs} ; \mathrm{N}=2$ for $\mathrm{hBO}$ s. e, Organoids continue to express pro-SFTPC (red) throughout culture (passage 5, left; passage 8, right), even following 9 months in culture, but do not exhibit expression of the AT1 marker HOPX (white) during later passages. DAPI (blue). Scale bar, $50 \mu \mathrm{M}$. f, Primary hAOs derived from hAT2 cells demonstrate expression of the AT1 markers HOPX (green, left) and PDPN (green, right) and the AT2 markers HTII-280 (red, left) and pro-SFTPC (red, right panel). DAPI (blue). Scale bar, $50 \mu \mathrm{m} . \mathbf{g}$, Immunofluorescent staining of ACE2 and TMPRSS2 (green) is consistent in hAOs derived from different donors. Scale bar, $50 \mu \mathrm{m}$. 
a

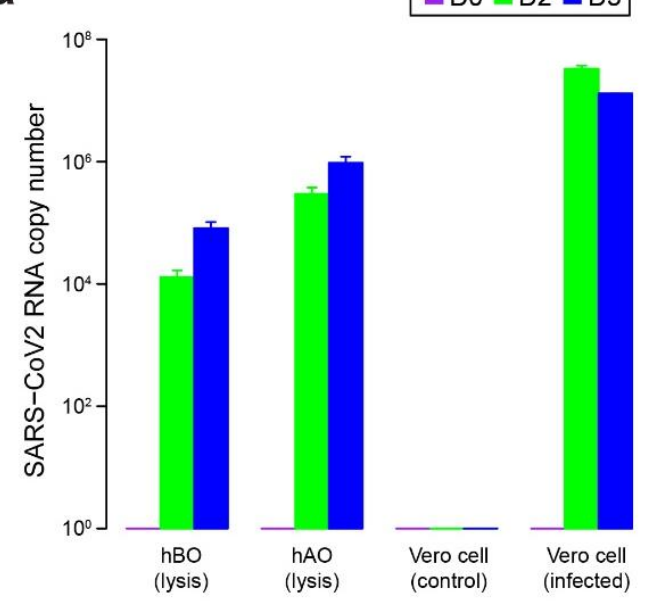

b
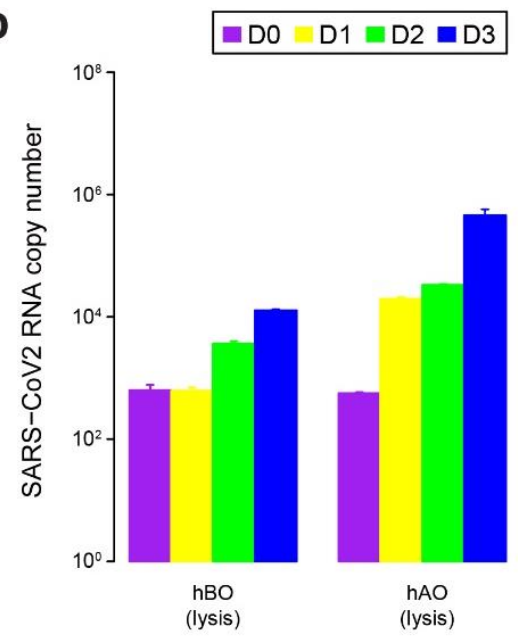

Extended Data Figure 2. Repeated experiments of SARS-CoV-2 infectivity in three-dimensional culture of human alveolar type 2 cells.

a, qPCR analysis for measuring the viral RNA levels in hBOs, hAOs, and vero cells (Replicate \#2). SARS-CoV-2 infectivity is much higher ( $<100$ times) in hAOs rather than hBOs. Error bars represent SEM. $n=2$. $\mathbf{b}, \mathrm{qPCR}$ analysis for measuring the viral RNA levels in hBOs and hAOs. (Replicate \#3). Error bars represent SEM. $n=3$ 
a
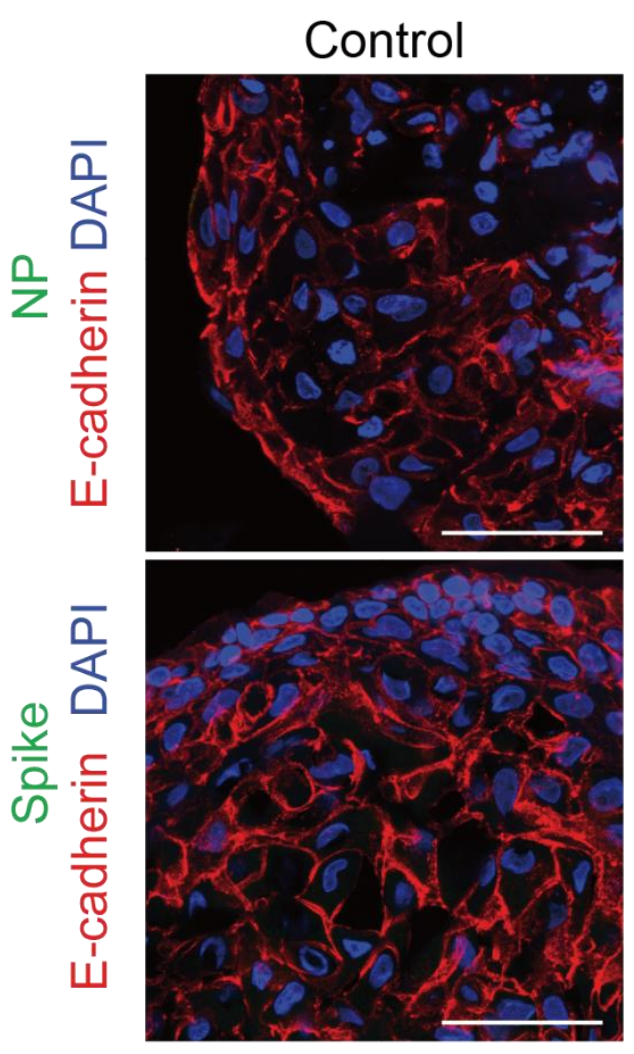

b

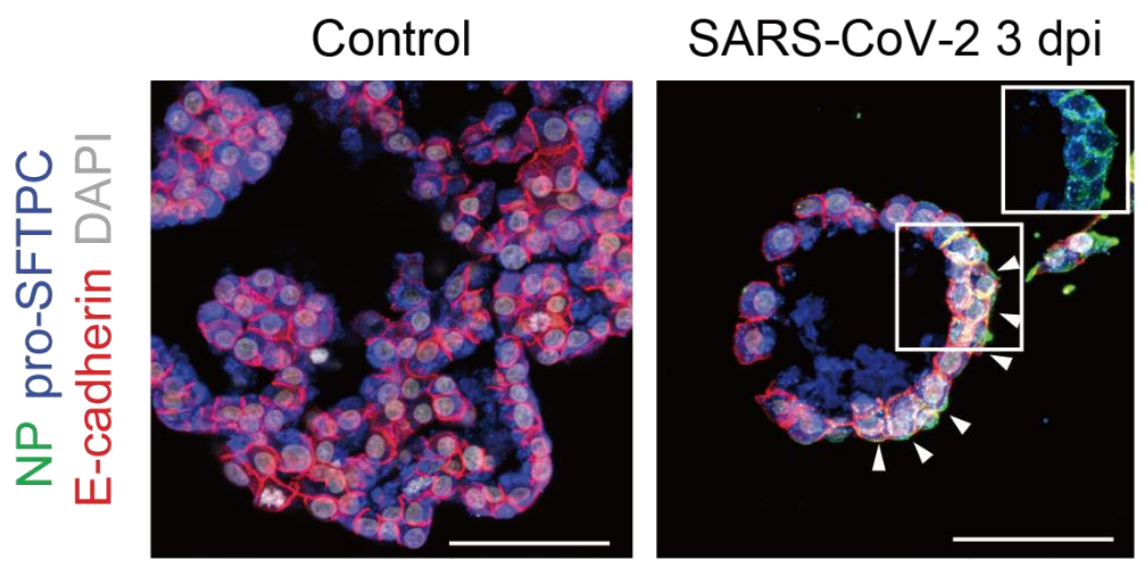

Extended Data Figure 3. Immunofluorescent images of human alveolar type 2 organoids at 3 days after SARS-CoV-2 infection.

a, In a portion of the hAO at $3 \mathrm{dpi}$, SARS-CoV-2 infection is identified by viral nucleoprotein (NP) and spike protein. Scale bar, $50 \mu \mathrm{m}$. b, SARS-CoV-2 infected hAOs still express the AT2 cell marker proSFTPC. Scale bar, $50 \mu \mathrm{m}$. 

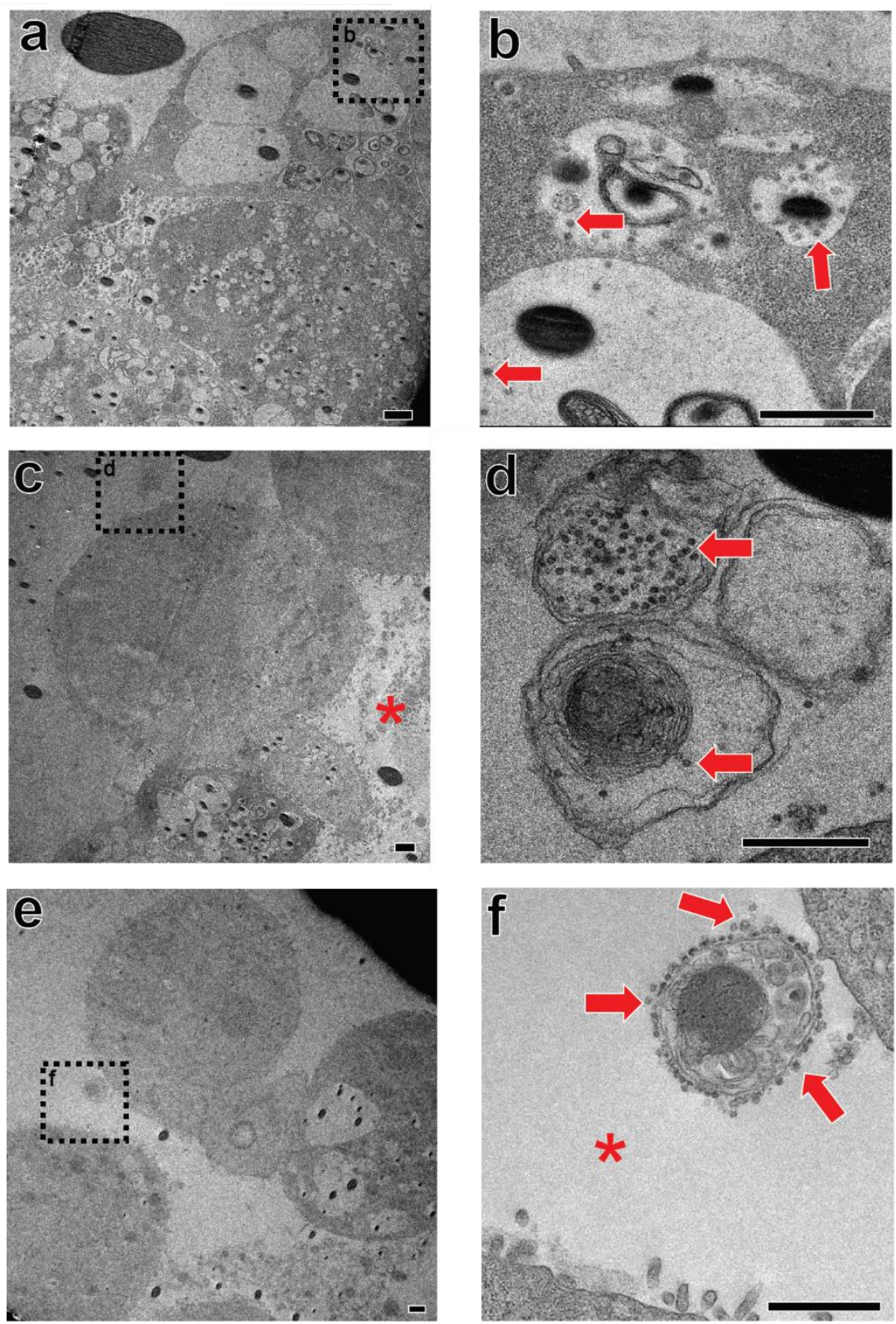

Extended Data Figure 4. Electron microscopy analysis of human alveolar type 2 cells infected

\section{by SARS-CoV-2.}

a, A low magnification image of an infected hAO. b, Viral particles (red arrow) in a large vacuole. The density of viral particles is lower than that of small vesicle structures. c, Another low magnification image of an infected hAO. Alveolar space is annotated by red asterisk. d, Viral particles are encapsulated in the exocytic vesicles (red arrow). e, A low magnification of hAT2 cells. f, Viral particles are attached to the outgoing vesicle (red arrow). Viruses are secreted in different ways. Scale bar, $1 \mu \mathrm{m}$. 
a

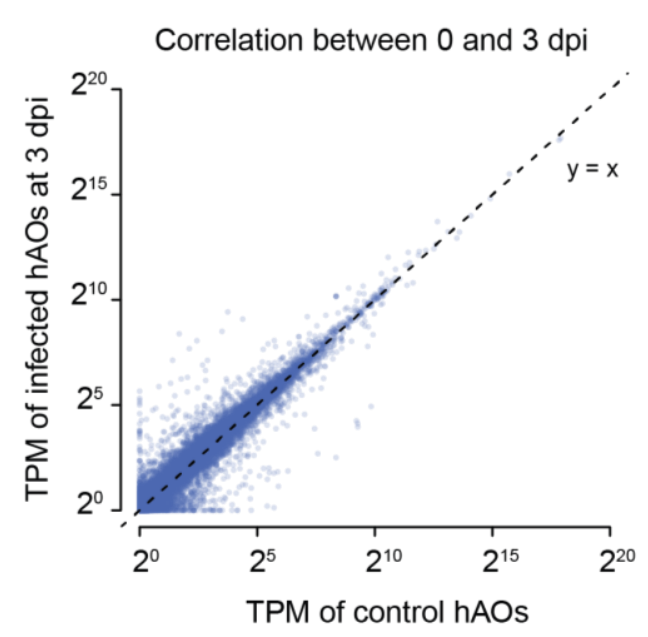

b

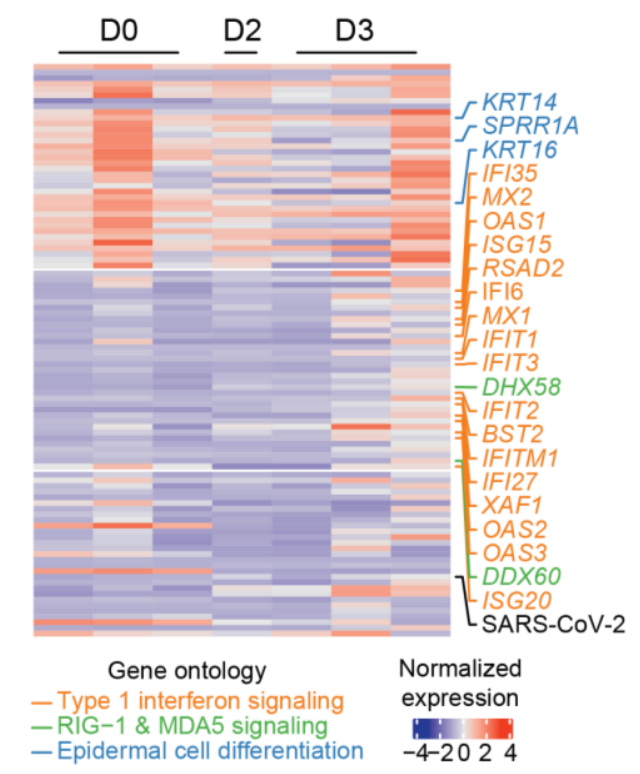

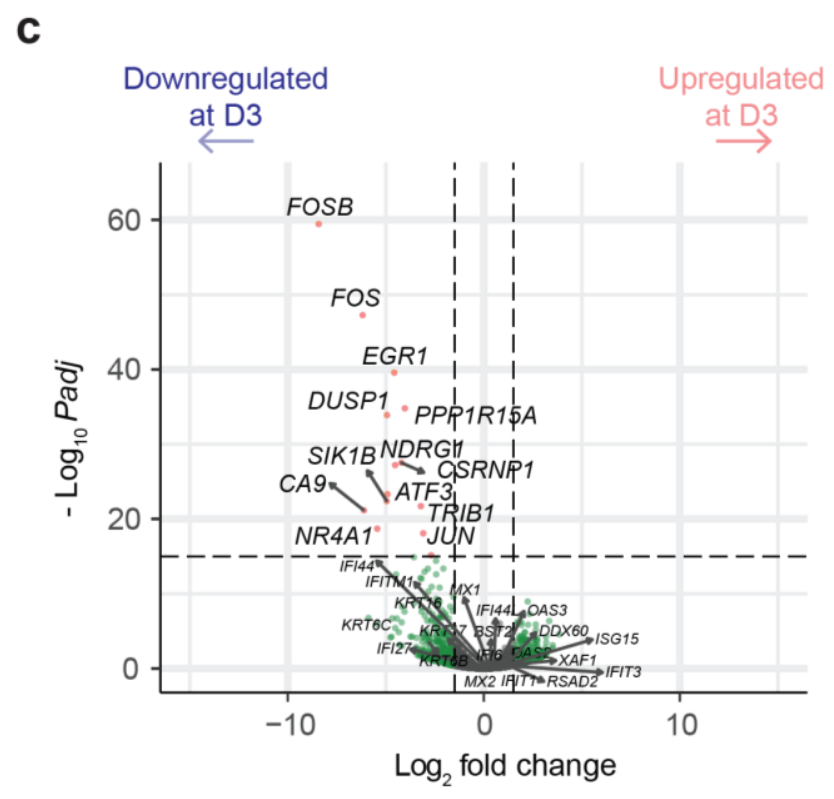

\section{Extended Data Figure 5. Transcriptome changes of the infected human lung organoids.}

a, Correlation of TPM level of each gene in hAOs at 0 and 3 dpi. Each gene is depicted as a blue dot. b, Heatmap of the most variable 100 genes derived from hAOs (Figure 5A, Table S1) in SARS-CoV-2 infected hBOs. Normalisation of gene expression was calculated using all transcriptome data from 14 samples of hAOs and hBOs. c, Volcano plot for differentially expressed genes in hBOs at 0 and 3 dpi. No significant changes were shown in ISGs. 
a

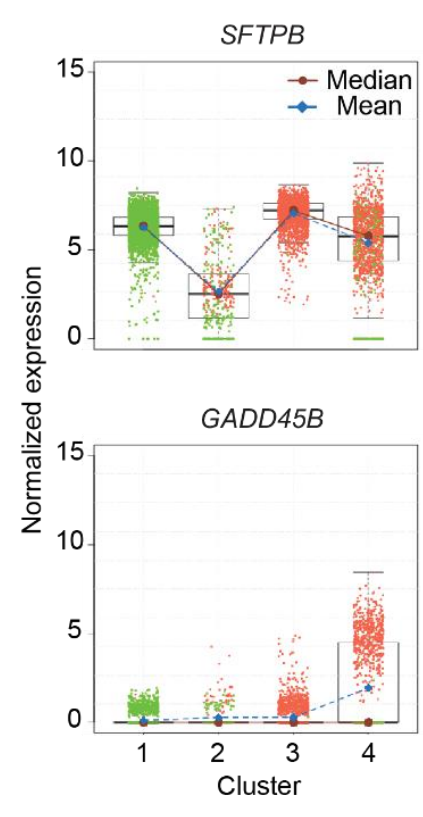

$N K X 2-1$

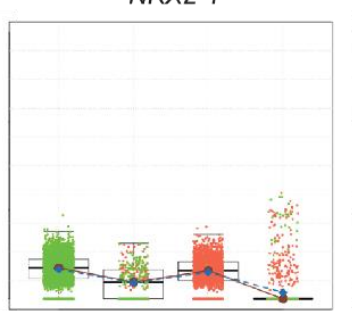

TNFAIP3

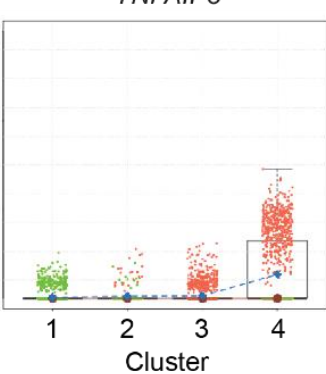

- Uninfected hAOs - Infected hAOs (D3)

b

SFTPC
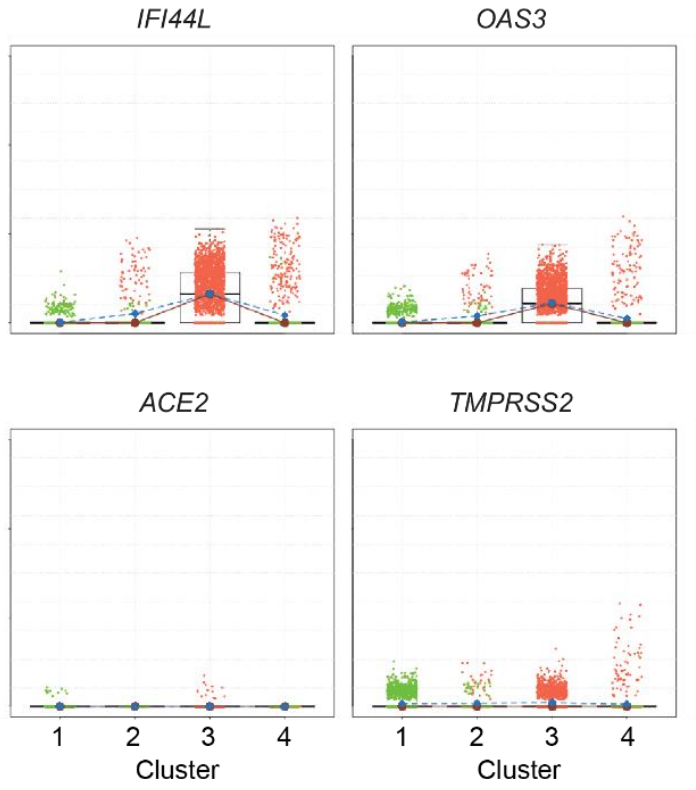

b
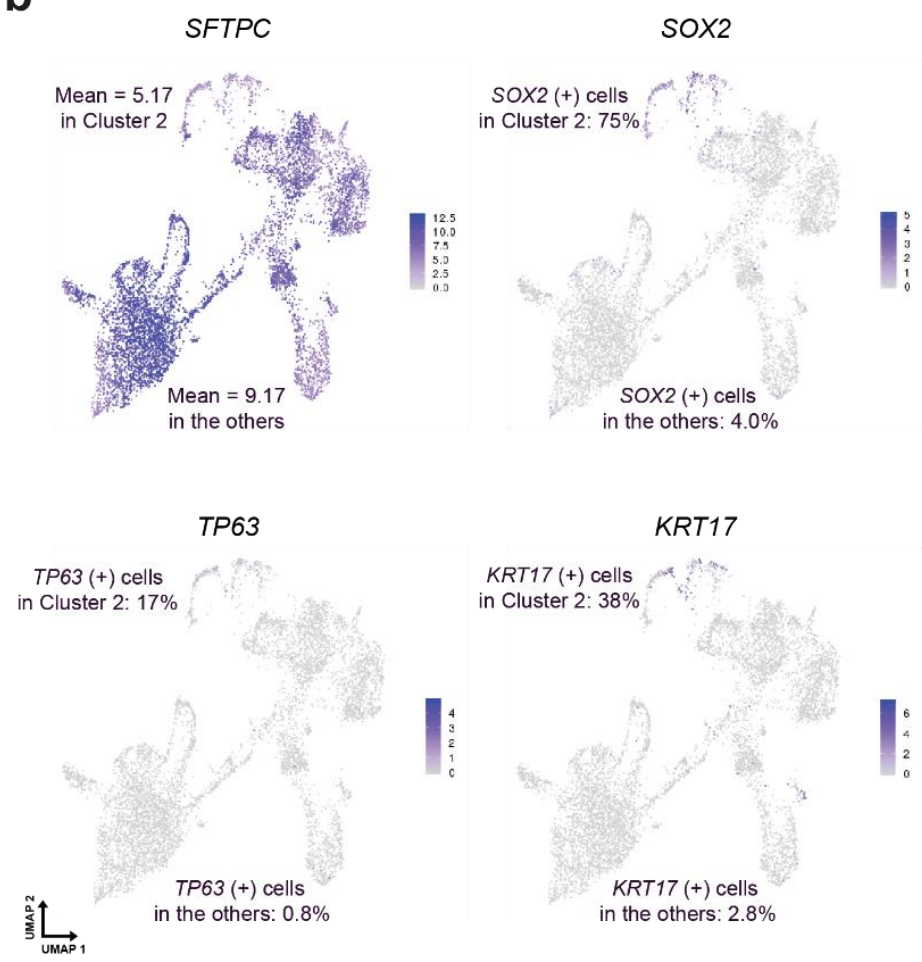

Extended Data Figure 6. Single-cell transcriptome profiling of infected hAOs.

a, Box plots showing normalised expression of interested genes in each cluster. Center line, median; box limits, upper and lower quartiles; whiskers, 1.5x interquartile range. b, In Cluster 2, expression level of SFTPC, one of hAT2 cell marker genes is reduced. By contrast, some airway marker genes such as SOX2, TP63, and KRT17 were expressed. 


\section{References}

1 Fung, T. S. \& Liu, D. X. Human Coronavirus: Host-Pathogen Interaction. Annu Rev Microbiol 73, 529-557, doi:10.1146/annurev-micro-020518-115759 (2019).

2 Wu, F. et al. A new coronavirus associated with human respiratory disease in China. Nature 579, 265-269, doi:10.1038/s41586-020-2008-3 (2020).

WHO. World Health Organization Coranavirus disease (COVID-19) Situation Report -140. (2020).

4 Muus, C. et al. Integrated analyses of single-cell atlases reveal age, gender, and smoking status associations with cell type-specific expression of mediators of SARS-CoV-2 viral entry and highlights inflammatory programs in putative target cells. bioRxiv (2020).

Hoffmann, M. et al. SARS-CoV-2 Cell Entry Depends on ACE2 and TMPRSS2 and Is Blocked by a Clinically Proven Protease Inhibitor. Cell 181, 271-280 e278, doi:10.1016/j.cell.2020.02.052 (2020).

6 Kim, D. et al. The Architecture of SARS-CoV-2 Transcriptome. Cell 181, 914-921 e910, doi:10.1016/j.cell.2020.04.011 (2020).

7 Walls, A. C. et al. Structure, Function, and Antigenicity of the SARS-CoV-2 Spike Glycoprotein. Cel/ 181, 281-292 e286, doi:10.1016/j.cell.2020.02.058 (2020).

8 Wang, Q. et al. Structural and Functional Basis of SARS-CoV-2 Entry by Using Human ACE2. Cell 181, 894-904 e899, doi:10.1016/j.cell.2020.03.045 (2020).

9 Chu, H. et al. Comparative tropism, replication kinetics, and cell damage profiling of SARSCoV-2 and SARS-CoV with implications for clinical manifestations, transmissibility, and laboratory studies of COVID-19: an observational study. The Lancet Microbe 1, e14-e23, doi:10.1016/S2666-5247(20)30004-5 (2020).

10 Bao, L. et al. The pathogenicity of SARS-CoV-2 in hACE2 transgenic mice. Nature, doi:10.1038/s41586-020-2312-y (2020).

11 Kim, Y. I. et al. Infection and Rapid Transmission of SARS-CoV-2 in Ferrets. Cell Host Microbe 27, 704-709 e702, doi:10.1016/j.chom.2020.03.023 (2020).

$12 \mathrm{Sia}, \mathrm{S}$. F. et al. Pathogenesis and transmission of SARS-CoV-2 in golden hamsters. Nature, doi:10.1038/s41586-020-2342-5 (2020).

13 Wolfel, R. et al. Virological assessment of hospitalized patients with COVID-2019. Nature 581, 465-469, doi:10.1038/s41586-020-2196-x (2020).

14 Andersen, K. G., Rambaut, A., Lipkin, W. I., Holmes, E. C. \& Garry, R. F. The proximal origin of SARS-CoV-2. Nat Med 26, 450-452, doi:10.1038/s41591-020-0820-9 (2020).

15 Forster, P., Forster, L., Renfrew, C. \& Forster, M. Phylogenetic network analysis of SARS-CoV2 genomes. Proc Natl Acad Sci U S A 117, 9241-9243, doi:10.1073/pnas.2004999117 (2020).

16 Shang, J. et al. Structural basis of receptor recognition by SARS-CoV-2. Nature 581, 221-224, doi:10.1038/s41586-020-2179-y (2020).

17 Fatehullah, A., Tan, S. H. \& Barker, N. Organoids as an in vitro model of human development and disease. Nat Cell Bio/ 18, 246-254, doi:10.1038/ncb3312 (2016). 
Heo, I. et al. Modelling Cryptosporidium infection in human small intestinal and lung organoids. Nat Microbio/3, 814-823, doi:10.1038/s41564-018-0177-8 (2018).

Bartfeld, S. et al. In vitro expansion of human gastric epithelial stem cells and their responses to bacterial infection. Gastroenterology 148, 126-136 e126, doi:10.1053/j.gastro.2014.09.042 (2015).

20 Elbadawi, M. \& Efferth, T. Organoids of human airways to study infectivity and cytopathy of SARS-CoV-2. Lancet Respir Med, doi:10.1016/S2213-2600(20)30238-1 (2020).

21 Lamers, M. M. et al. SARS-CoV-2 productively infects human gut enterocytes. Science, doi:10.1126/science.abc1669 (2020).

22 Monteil, V. et al. Inhibition of SARS-CoV-2 Infections in Engineered Human Tissues Using Clinical-Grade Soluble Human ACE2. Cel/ 181, 905-913 e907, doi:10.1016/j.cell.2020.04.004 (2020).

23 Yang, L. et al. A Human Pluripotent Stem Cell-based Platform to Study SARS-CoV-2 Tropism and Model Virus Infection in Human Cells and Organoids. Cell Stem Cel/ 27, 125-136 e127, doi:10.1016/j.stem.2020.06.015 (2020).

24 Barkauskas, C. E. et al. Lung organoids: current uses and future promise. Development 144, 986-997, doi:10.1242/dev.140103 (2017).

25 Barkauskas, C. E. et al. Type 2 alveolar cells are stem cells in adult lung. J Clin Invest 123, 3025-3036, doi:10.1172/Jci68782 (2013).

26 Zacharias, W. J. et al. Regeneration of the lung alveolus by an evolutionarily conserved epithelial progenitor. Nature 555, 251-255, doi:10.1038/nature25786 (2018).

27 Kathiriya, J. J. et al. Human alveolar Type 2 epithelium transdifferentiates into metaplastic KRT5 + basal cells during alveolar repair. bioRxiv (2020).

28 Gonzalez, R. F., Allen, L., Gonzales, L., Ballard, P. L. \& Dobbs, L. G. HTII-280, a biomarker specific to the apical plasma membrane of human lung alveolar type II cells. J Histochem Cytochem 58, 891-901, doi:10.1369/jhc.2010.956433 (2010).

29 Nikolic, M. Z. et al. Human embryonic lung epithelial tips are multipotent progenitors that can be expanded in vitro as long-term self-renewing organoids. Elife 6, doi:10.7554/eLife.26575 (2017).

30 Sachs, N. et al. Long-term expanding human airway organoids for disease modeling. EMBO J 38, doi:10.15252/embj.2018100300 (2019).

31 Kim, J. M. et al. Identification of Coronavirus Isolated from a Patient in Korea with COVID19. Osong Public Health Res Perspect 11, 3-7, doi:10.24171/j.phrp.2020.11.1.02 (2020).

32 Suzuki, T. et al. Generation of human bronchial organoids for SARS-CoV-2 research. bioRxiv (2020).

33 Knoops, K. et al. SARS-coronavirus replication is supported by a reticulovesicular network of modified endoplasmic reticulum. PLoS Bio/6, e226, doi:10.1371/journal.pbio.0060226 (2008).

34 Arbour, N. et al. Acute and persistent infection of human neural cell lines by human coronavirus OC43. J Virol 73, 3338-3350 (1999). 
Maier, H. J. et al. Infectious bronchitis virus generates spherules from zippered endoplasmic reticulum membranes. mBio 4, e00801-00813, doi:10.1128/mBio.00801-13 (2013). Ogando, N. S. et al. SARS-coronavirus-2 replication in Vero E6 cells: replication kinetics, rapid adaptation and cytopathology. bioRxiv (2020).

37 Iwasaki, A., Foxman, E. F. \& Molony, R. D. Early local immune defences in the respiratory tract. Nat Rev Immuno/ 17, 7-20, doi:10.1038/nri.2016.117 (2017).

38 Schneider, W. M., Chevillotte, M. D. \& Rice, C. M. Interferon-stimulated genes: a complex web of host defenses. Annu Rev Immuno/ 32, 513-545, doi:10.1146/annurev-immunol032713-120231 (2014).

39 Forero, A. et al. Differential Activation of the Transcription Factor IRF1 Underlies the Distinct Immune Responses Elicited by Type I and Type III Interferons. Immunity 51, 451-464 e456, doi:10.1016/j.immuni.2019.07.007 (2019).

40 Park, A. \& Iwasaki, A. Type I and Type III Interferons - Induction, Signaling, Evasion, and Application to Combat COVID-19. Cell Host Microbe, doi:10.1016/j.chom.2020.05.008 (2020).

41 Ziegler, C. G. K. et al. SARS-CoV-2 Receptor ACE2 Is an Interferon-Stimulated Gene in Human Airway Epithelial Cells and Is Detected in Specific Cell Subsets across Tissues. Cel/ 181, 10161035 e1019, doi:10.1016/j.cell.2020.04.035 (2020).

42 Blanco-Melo, D. et al. Imbalanced Host Response to SARS-CoV-2 Drives Development of COVID-19. Cel/ 181, 1036-1045 e1039, doi:10.1016/j.cell.2020.04.026 (2020).

43 Lee, J. H. et al. Lung stem cell differentiation in mice directed by endothelial cells via a BMP4-NFATc1-thrombospondin-1 axis. Cell 156, 440-455, doi:10.1016/j.cell.2013.12.039 (2014).

44 Fujii, M. et al. Human Intestinal Organoids Maintain Self-Renewal Capacity and Cellular Diversity in Niche-Inspired Culture Condition. Cell Stem Cell 23, 787-793 e786, doi:10.1016/j.stem.2018.11.016 (2018).

$45 \mathrm{Kim}$, J. H. et al. Clinical diagnosis of early dengue infection by novel one-step multiplex realtime RT-PCR targeting NS1 gene. J Clin Viro/65, 11-19, doi:10.1016/j.jcv.2015.01.018 (2015). Dobin, A. et al. STAR: ultrafast universal RNA-seq aligner. Bioinformatics 29, 15-21, doi:10.1093/bioinformatics/bts635 (2013).

47 Li, B. \& Dewey, C. N. RSEM: accurate transcript quantification from RNA-Seq data with or without a reference genome. BMC Bioinformatics 12, 323, doi:10.1186/1471-2105-12-323 (2011).

48 Love, M. I., Huber, W. \& Anders, S. Moderated estimation of fold change and dispersion for RNA-seq data with DESeq2. Genome Bio/ 15, 550, doi:10.1186/s13059-014-0550-8 (2014).

49 Saunders, C. T. et al. Strelka: accurate somatic small-variant calling from sequenced tumornormal sample pairs. Bioinformatics 28, 1811-1817, doi:10.1093/bioinformatics/bts271 (2012).

50 Koboldt, D. C. et al. VarScan 2: somatic mutation and copy number alteration discovery in cancer by exome sequencing. Genome Res 22, 568-576, doi:10.1101/gr.129684.111 (2012). 

made available under aCC-BY-NC-ND 4.0 International license.

51 Li, H. et al. The Sequence Alignment/Map format and SAMtools. Bioinformatics 25, 20782079, doi:10.1093/bioinformatics/btp352 (2009).

52 Robinson, J. T. et al. Integrative genomics viewer. Nat Biotechnol 29, 24-26, doi:10.1038/nbt.1754 (2011).

53 Stuart, T. et al. Comprehensive Integration of Single-Cell Data. Cell 177, 1888-1902 e1821, doi:10.1016/j.cell.2019.05.031 (2019).

54 Jensen-Taubman, S. M., Steinberg, S. M. \& Linnoila, R. I. Bronchiolization of the alveoli in lung cancer: pathology, patterns of differentiation and oncogene expression. Int J Cancer 75, 489-496, doi:10.1002/(sici)1097-0215(19980209)75:4<489::aid-ijc1>3.0.co;2-p (1998).

$55 \mathrm{Xu}, \mathrm{Y}$. et al. Single-cell RNA sequencing identifies diverse roles of epithelial cells in idiopathic pulmonary fibrosis. JCl Insight 1, e90558, doi:10.1172/jci.insight.90558 (2016). 